Wilfried E. Keil

\title{
Korrelationen zwischen kommunalen Inschriften und Bauskulpturen im mittelalterlichen Ober- und Mittelitalien
}

\author{
Fallbeispiele in Genua, Mailand und Montefalco
}

Die Verbindung zwischen Inschriften und Bauskulpturen kommt im kommunalen Bereich weit weniger vor als im sakralen Umfeld. Bei kommunalen Inschriften ist der Zusammenhang mit Skulpturen besonders häufig in Genua anzutreffen. ${ }^{1} \mathrm{Ob}$ dies nur an der Überlieferungssituation liegt oder ob es sich hierbei um eine Eigenart der Kommune von Genua handelt, konnte bisher nicht geklärt werden. Allerdings ist auch zu bedenken, dass zu diesem Thema kaum eingehende Untersuchungen vorliegen. In dieser Studie wird daher nicht nur ein Beispiel aus Genua behandelt, sondern auch eines aus einer anderen großen Kommune, Mailand, und eines aus Montefalco, einer kleinen Kommune in Mittelitalien. Zudem sind die Beispiele so gewählt, dass unterschiedliche Faktoren untersucht werden können. Das Material der Artefakte ist Stein bzw. Marmor. Dieses Material wurde von den Kommunen bewusst aufgrund seiner Eigenschaften wie Beständigkeit, Dauerhaftigkeit und Widerstandsfähigkeit gewählt, da hierdurch die Präsenz des Artefakts auch mit diesen Eigenschaften auf den Rezipienten wirkt. Das Material wird aufgrund des Inhalts der Inschriften gewählt. Diese sind von dauerhafter Bedeutung und sollen in der Erinnerungskultur langfristig präsent bleiben. ${ }^{2}$ Ein anderer Aspekt ist wohl der bewusste Bezug auf die Tradition der Verwendung des Materials von Herrschern und anderen Machtträgern. Die Bildwirkung von Skulpturen ${ }^{3}$ und die Schriftwirkung ${ }^{4}$ im Mittelalter wurde immer wieder untersucht, aber die spezifischen Merkmale der Kombination von Skulptur und Inschriften nicht eingehend. ${ }^{5}$

\footnotetext{
$1 \mathrm{Zu}$ weiteren Beispielen aus Genua siehe den Beitrag von Rebecca Müller in diesem Band.

2 Zur Materialität von Stein als Schriftträger und Stein als Text- und Schriftträger siehe Balke et al. 2015, 248-252, 256f. Zu den Materialeigenschaften von Stein im Hinblick auf Inschriften siehe auch Favreau 1997, 47f.

3 Siehe z. B. Boerner 2008.

4 Siehe z. B. Debiais 2009. Zur kommunalen Epigraphik in Städten im Allgemeinen siehe Giovè Marchioli 1994.

5 Untersuchungen zum Verhältnis von Bild und Text im Mittelalter gibt es zahlreiche; diese behandeln allerdings meistens die Buchmalerei. Siehe hierzu z. B.: Saurma-Jeltsch 1988; Curschmann 2007;

Dieser Beitrag ist im Heidelberger Sonderforschungsbereich 933 „Materiale Textkulturen. Materialität und Präsenz des Geschriebenen in non-typographischen Gesellschaften“ entstanden. Der SFB 933 wird durch die Deutsche Forschungsgemeinschaft finanziert.
}

Ә Open Access. (c) 2019 Wilfried E. Keil, publiziert von De Gruyter. (cc) BY-NC-ND Dieses Werk ist lizenziert unter der Creative Commons Attribution-NonCommercial-NoDerivatives 4.0 Lizenz. 
Es fragt sich, inwieweit die Schriftwirkung durch das Bildwerk und umgekehrt die Bildwirkung durch die Schrift beeinflusst wird. Erzeugt die Kombination für den Betrachter eine zusätzliche Wirkung oder nicht? Die Seltenheit einer Kombination von Schrift und Bild bei kommunalen Inschriften lässt auf eine bewusste Intention schließen. Wie wirkte diese auf den Rezipienten?

\section{Die Löwen am Palazzo di San Giorgio in Genua}

Am Hafen von Genua liegt der Palazzo di San Giorgio, der ehemalige Palazzo Comunale, auch Palazzo del Mare und Palazzo del Capitano genannt. Der Gebäudekomplex wurde ab 1260 als Kommunalpalast gebaut. ${ }^{6}$ Er war Amtssitz der Magistrate und somit ein wichtiger Repräsentationsbau. Bevor dem Rat ein eigenes Bauwerk errichtet wurde, tagte er in kirchlichen Gebäuden oder Adelspalästen im Umkreis der Kathedrale. ${ }^{7}$ Die Funktion eines Kommunalpalastes spiegelt sich auch in seiner Ausstattung wieder, zu der neben Wandmalereien von Kampfszenen auch Trophäen gehörten. ${ }^{8}$ Die hier behandelte Trophäe befindet sich am Portal, das von der Portikus zum Innenhof führt. Nach seiner Erbauung behielt das Gebäude zunächst seine Funktion unter den Guelfen und dann auch unter dem ghibellinischen Doppelkapitanat. ${ }^{9} 1291$ wurde für die Stadt ein Palast in der Nähe der Kathedrale errichtet. ${ }^{10}$ Der alte Kommunalpalast diente dann anderen administrativen Aufgaben wie dem Sitz des Zolls und anderer Behörden und wurde palatium duganae maris, palatium de mare oder dugana genannt. ${ }^{11}$ Ab 1451 wurde das Bauwerk der Sitz der städtischen Bank Società delle Compere e dei Banchi di San Giorgio. ${ }^{12}$ Seither wird das Gebäude Palazzo di San Giorgio genannt. Ab 1570/1571 wurde der Palast durch große Erweite-

Krause/Schellewald 2011. Von den bisherigen Untersuchungen zum Verhältnis von Skulptur und Inschriften seien folgende beispielhaft genannt: Verzar 1994; Späth 2011. Zum Verhältnis von Skulptur und Inschriften im kommunalen Bereich ist von Hülsen-Esch 1994 hervorzuheben.

6 Dietl 2009, Bd. 2, 900. Zum Palazzo S. di Giorgio im Allgemeinen siehe: Paul 1963, 226-228; Rotondi Terminiello 1977; Grosso 1984; Cavallaro 1992; Ferrando Cabona 1998.

7 Grossi Bianchi/Poleggi 1987, 106, 109.

8 Paul 1963, 117-121; Müller 2002, 86. Zu Trophäen im Allgemeinen siehe: Müller 2002, 47-57. Zu Trophäen in Genua siehe: Müller 2002; Müller 2005; Haug 2016, 193-288.

9 Müller 2002, 89f.; Dietl 2009, Bd. 2, 900.

10 Cavallaro 1992, 53; Müller 2002, 90. Dort ältere Literatur hierzu in Anm. 245.

11 Paul 1963, 227; Grosso 1984, 18; Cavallaro 1992, 35, 53; Ferrando Cabona 1998, 34; Müller 2002, 90. 12 Rotondi Terminiello 1977, 15; Grosso 1984, 6; Cavallaro 1992, 54; Ferrando Cabona 1998, 35, 90. Die Bank wurde durch ein Dekret der französischen Republik am 15. Dezember 1797 aufgelöst. Siehe Armillotta 2009/10, 121. Danach wurde der Palast teilweise privat genutzt. 1822 kam es zu der Idee im Rahmen einer Neugestaltung des Hafengebietes die Portikus des 13. Jahrhunderts abzureißen. Dies konnte zwischenzeitlich verhindert, aber erst 1889 durch die Commissione Conservatrice dei Мonumenti endgültig abgewendet werden. Siehe hierzu Armillotta 2009/10, $121 f$. 
rungsbauten in Richtung Meer grundlegend umgestaltet. ${ }^{13}$ Hierbei wurde die Portikus wohl bewusst nicht umgebaut oder ummantelt, sondern blieb in ihrer ursprünglichen Gestalt bestehen. Von 1891 bis 1905 fanden umfangreiche Restaurierungsmaßnahmen durch Alfredo d'Andrade statt. ${ }^{14}$ Seit dem 29. Oktober 1905 war der Palast der Sitz des Consorzio Autonomo del Porto. ${ }^{15}$ Im Oktober 1942 und im August 1944 kam es durch Fliegerbomben zu großen Zerstörungen in Genua. Hierbei wurde auch der Palazzo beschädigt, ${ }^{16}$ allerdings nicht an der Portikus, sodass an diesem Bauteil die Situation bei den darauf folgenden Restaurierungen wohl nicht verändert wurde. ${ }^{17}$

Der Palazzo war ursprünglich ein dreigeschossiger Bau mit longitudinalem Grundriss. Die Schmalseite im Westen zeigte Richtung Meer. An der noch heute erhaltenen Ostfassade, die zum Stadtinneren gerichtet ist, hat er eine fünfachsige Portikus. Beides befindet sich weitestgehend im Originalzustand. ${ }^{18}$ Alfredo d'Andrade hat bei der Restaurierung Ende des 19. Jahrhunderts den Zinnenkranz erneuert und Teile der Seitenwände restauriert. Die Säulen wurden um 1500 ersetzt. ${ }^{19}$ In der Flucht der mittleren Arkade steht das Hauptportal. Oberhalb des Portals zum Innenhof ist im Lünettenfeld unter einer Bauinschrift eine Löwenkopfskulptur in ungefähr fünf Metern Höhe eingemauert (Abb. 1 und 2). Der Türsturz ruht auf Kragsteinen. Darüber befindet sich ein leicht spitzförmiger Bogen, der abwechselnd aus grauem Kalkstein und Marmor gefertigt wurde. Vom Bogen zum Lünettenfeld vermitteln profilierte Rippen, die wahrscheinlich aus Marmor bestehen. Das Lünettenfeld selbst ist aus vier Steinlagen gemauert. Die unterste ist ein großer monolither Kalkstein, in dessen Mitte unterhalb des Löwenkopfes ein leicht erhabener Wappenschild herausragt. In den Schild ist das Stadtwappen von Genua, ein rotes lateinisches Kreuz auf weißem Grund, gemalt. Die zweite Steinlage wurde aus Marmor gesetzt. In der Mitte ist die

13 Paul 1963, 228; Rotondi Terminiello 1977, 9f.; Grosso 1984, 10, 26; Cavallaro 1992, 54, 81; Ferrando Cabona 1998, 28, 31, 100. Die Außenseite wurde von 1606 bis 1608 von Lazzaro Tavarone bemalt. Siehe Rotondi Terminiello 1977, 10; Grosso 1984, 26; Caravallo 1992, 84. Diese Malereien wurden dann 1912 von Ludovico Pogliaghi nachgemalt. Siehe Rotondi Terminiello 1977, 14; Grosso 1984, 26.

14 Rotondi Terminiello 1977, 14. Zur Restaurierung siehe ebd., 10-15. Im März 1889 wurde in Vorbereitung zu den Restaurierungsmaßnahmen eine Kommission eingesetzt. Siehe Boito 1893, 273. Zu Alfredo D’Andrade siehe Marcenaro 1990.

15 Cavallaro 1992, 93. Seit dem 1. Januar 1995 ist der Palazzo di San Giorgio Sitz der Autorità Portuale di Genova. Siehe: Ferrando Cabona 1998, 20.

16 Ferrando Cabona 1998, 20. Genaueres hierzu bei: Armillotta 2009/10, 122. Zu der Organisation der Restaurierung und den damit verbundenen finanziellen Schwierigkeiten siehe Armillotta 2009/10, 122-128.

17 Die Portikus und damit auch das Portal scheinen nach alten Aufnahmen durch die Bomben nicht groß in Mitleidenschaft gekommen zu sein. Ich danke Dott. Mario Caselli, dem Leiter des historischen Archivs der Autorità Portuale di Genova, für die freundliche Auskunft.

18 Müller 2002, 86.

19 Ebd., 86 Anm. 219. Zu Untersuchungen und Restaurierungen des gesamten Palastes siehe Cavallaro $1992,79-137$. 


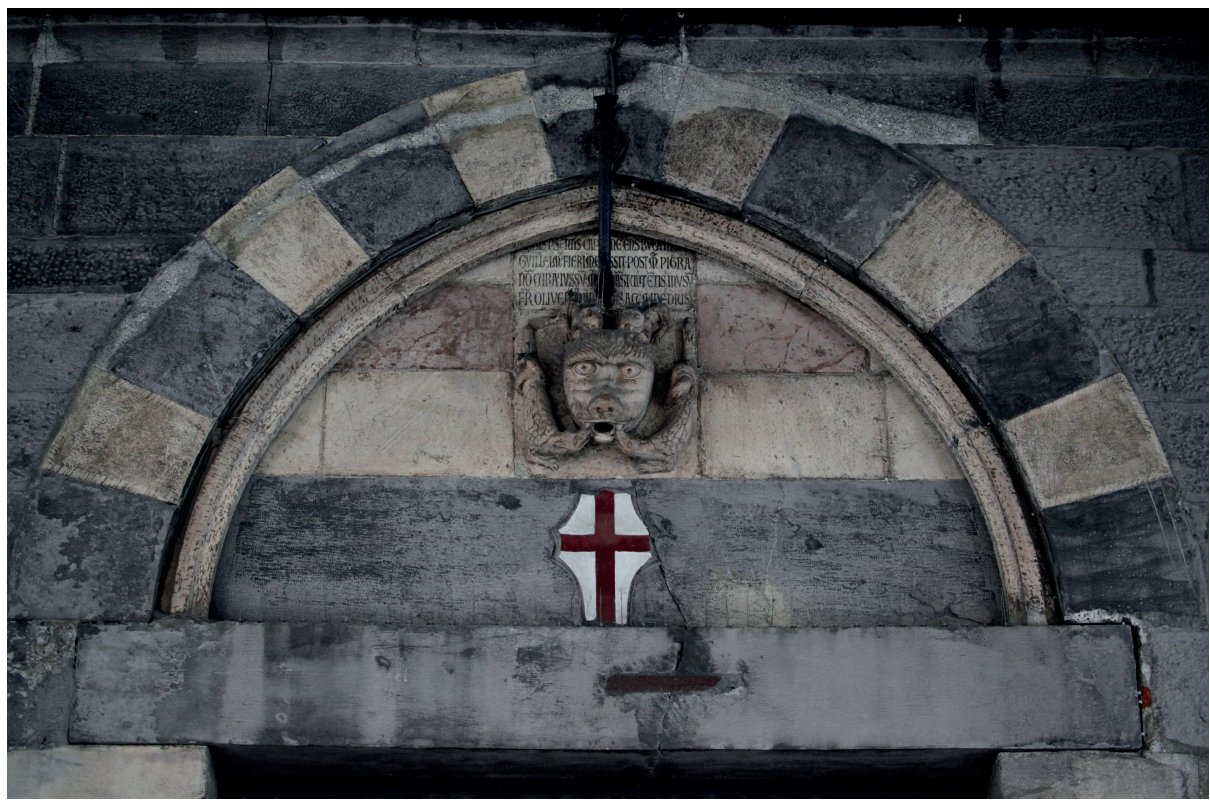

Abb. 1: Genua; Palazzo di San Giorgio, Ostfassade, Hauptportal, Lünettenfeld mit Löwenkopf und Inschrift (Foto @ Wilfried E. Keil).

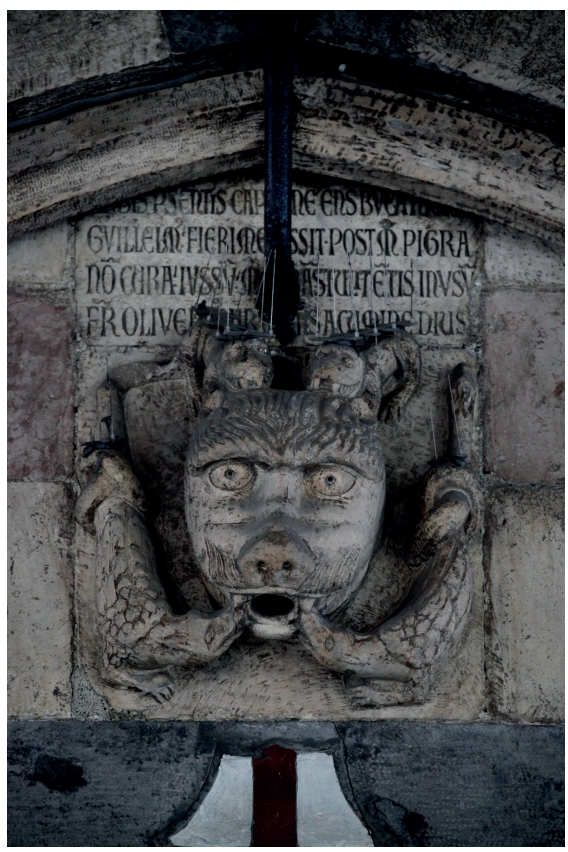

Abb. 2: Genua; Palazzo di San Giorgio, Ostfassade, Hauptportal, Löwenkopf und Inschrift (Foto @ Wilfried E. Keil). 
Löwenkopfskulptur vermauert, die in die darüber liegende Steinlage hineinragt. Die dritte Steinlage besteht aus zwei rosafarbenen Steinen. In der Mitte über dem Löwenkopf steht bündig eine Inschriftentafel, die auch die vierte Lage einnimmt, die aus hellem Marmor gebildet ist.

Die Skulptur ist aus einem nahezu quadratischen bräunlichen Marmorblock ${ }^{20}$ mit einer Höhe von 45 Zentimetern und eine Breite von 50 Zentimetern gehauen. ${ }^{21}$ Der Löwenkopf besticht durch seine großen Augen und seine große Nase. Sowohl die Mähne als auch der Oberlippenbart sind stilisiert ausgearbeitet. Der Löwe hat das Maul leicht geöffnet, sodass ein Teil der Vorderzähne sichtbar ist. In der Mitte des Mauls ist ein großes rundes Loch, das früher wahrscheinlich als Wasserauslauf diente. Der Löwenkopf hatte also einmal die Funktion eines Wasserspeiers oder eines Brunnenauslaufes. Auf dem Kopf des Löwen stehen zwei kleine Löwen, die ihre Zähne blecken. Bei genauer Betrachtung fällt auf, dass sie nur ihre Unterkiefer und ihre Vorderläufe auf den großen Löwenkopf gestützt haben. Mit ihren Hinterläufen stützen sie sich an der Steinwand ab. Man könnte zunächst meinen, dass die kleinen Löwen sich in der Mähne des großen Löwen verbeißen. Dies ist aber nicht der Fall. Neben und unterhalb des Löwenkopfs befindet sich auf jeder Seite jeweils ein Drache mit zwei Beinen und verknotetem Schwanz. Ihre Vorderkörper haben sie so gedreht, dass sie ihre Mäuler zu dem des Löwen gerichtet haben. Es ist nicht genau ersichtlich, ob sie den Löwen in die Unterlippe beißen wollen oder ob sie nur ihre Mäuler dürstend in Richtung Wasserauslauf halten. Beim linken Drachen ist das Schwanzende beschädigt. Der Schwanz des rechten Drachen endet in einem kleinen Drachenkopf. Die Inschriftentafel ist links und rechts bündig mit dem Block des Löwenkopfes vermauert. Die oberste Zeile der Inschrift wird nahezu komplett und die zweite Zeile teilweise durch die Rippen verdeckt. Zwischen der vorletzten und letzten Schriftzeile ist ein Metallstab mit Blei in den Stein eingelassen. Dieser scheint heute als Blitzableiter zu dienen. Die Inschrift weist also eine eingeschränkte Sichtbarkeit auf. Sie hat somit eine restringierte Schriftpräsenz. ${ }^{22}$ Im September 1873 wurde der Stein bei einer Restaurierung durch Alfredo d'Andrade abgenommen und so konnten auch die sonst nicht sichtbaren Teile der Inschrift dokumentiert werden. ${ }^{23}$

Die Inschrift ist fünfzeilig und in gotischer Majuskel geschrieben (Abb. 3). Sie besteht aus leoninischen Hexametern.

20 Dietl 2009, Bd. 2, 899.

21 Müller 2002, 214; Dietl 2009, Bd. 2, 899.

22 Zu Bauinschriften mit restringierter Präsenz siehe Keil 2014. Zur restringierten Schriftpräsenz im Allgemeinen siehe Frese/Keil/Krüger 2014.

23 Remondini 1874, 93; Boito 1893, 276, 28; Cavallaro 1992, 34 Anm. 3; Ferrando Cabona 1998, 44. Wahrscheinlich hat Marcello Remondini die Inschrift 1874 erstmals vollständig publiziert. 


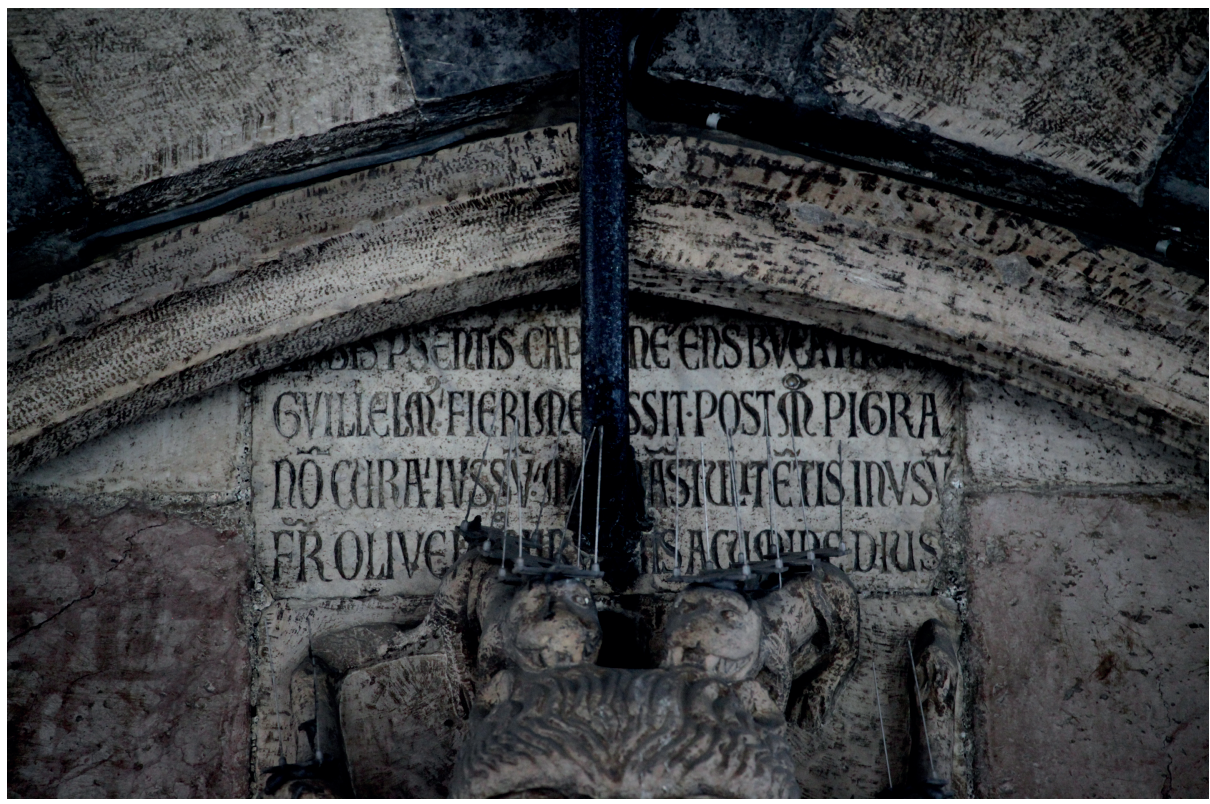

Abb. 3: Genua; Palazzo di San Giorgio, Ostfassade, Hauptportal, Inschrift (Foto (C Wilfried E. Keil).

\author{
[ANNO MILLENO BISCENTVM DECIES QVOQVE SENO] / \\ VRBIS P(RE)SENTIS CAPITANE(VS) E(XISTE)NS BVCANIGRA / \\ GVILLELM(VS) FIERI ME IVSSIT POSTM(OD)O PIGRA/ \\ NO(N) CVRA IVSSV(M) M(E) TRA(N)STVLIT ET(ERN)IS IN VSV(M)/ \\ FR(ATER) OLIVER(IVS) VIR ME(N)TIS ACV(M)I(N)E DIV(V)S
}

Im Jahr 1260 hat mich Guglielmo Boccanegra, als er Capitan der gegenwärtigen Stadt war, anzufertigen befohlen. Schließlich hat mich, in diesem Auftrag, Frater Oliverius, der durch die Scharfsinnigkeit seines Geistes gesegnete Mann, mit nicht nachlassender Sorge in den Zustand der Nutzbarkeit für ewige Zeiten (?) überführt. ${ }^{24}$

Die Inschrift ist trotz einiger Kürzungen, die gekennzeichnet sind, recht leicht zu lesen.

Aus der Bauinschrift geht hervor, dass der Bau vom Capitano del popolo Guglielmo Boccanegra 1260 in Auftrag gegeben wurde und dass ein Frater Oliverius den Bau ausgeführt hat. Der vor 1274 gestorbene Guglielmo Boccanegra stammte aus einer reichen Genueser Popularenfamilie. ${ }^{25}$ Nach Volksaufständen wurde er 1257 zum Capitano del popolo von Genua erhoben. Weiter ist über ihn aus Schriftquellen bekannt,

24 Übersetzung nach Albert Dietl; zur Inschrift siehe Dietl 2009, Bd. 2, 899. Siehe auch Silva 1987, 109 Nr.190. Zur wohl erstmaligen Wiedergabe der gesamten Inschrift siehe Remondini 1874, 93. Zu einer frühen Publikation ohne den verdeckten Anfang der Inschrift siehe Cuneo 1842, 197.

25 Dietl 2009, Bd. 2, 899. Siehe auch Cavallaro 1992, 37. 
dass er im März 1261 für Genua Handelsprivilegien mit Konstantinopel ausgehandelt hat. Nachdem ihm das venezianische Viertel Konstantinopels übergeben wurde, ließ er den venezianischen Palast zerstören. In einem Aufruhr Anfang Mai 1262 wurde er in Genua abgesetzt. ${ }^{26} \mathrm{Am}$ 9. Mai 1262 musste er ins Exil gehen und trat in die Dienste der französischen Könige Ludwig IX. (1226-1270) und Philipp III. (1270-1285) als Kommandant der Festung von Aigues-Mortes. ${ }^{27}$ Der Zisterzienserbruder Oliverius ist für die Jahre 1239 bis 1255 im Genueser Zisterzienserkloster S. Andrea di Sestri belegt. ${ }^{28}$ Zwischen 1257 und 1260 ist er in vier Urkunden als minister et operarius operis portus et moduli civitatis Ianue belegt. Er war also mindestens für die Beschaffung des Baumaterials zuständig. ${ }^{29}$ Allerdings ist seine Rolle bei den Bauarbeiten umstritten. Einige benennen ihn korrekt als Bauverwalter, ${ }^{30}$ andere wollen in ihm sogar auch den Architekten sehen. ${ }^{31}$ Frater Oliverius errichtete ab 1260 ein erstes autonomes Kommunalgebäude für den Genueser Magistrat, das Palatium comunis Ianue de Ripa. ${ }^{32}$ Dass das Gebäude recht früh benutzt werden konnte, ist durch einen Notariatsakt vom 18. Mai 1260 belegt, der in dem gerade im Bau befindlichen Palast vollzogen wurde. Dieser bezeugt einen Vertrag für Baumaterial für den Hafen mit Frater Oliverius, dem Bauverwalter des Kommunalpalastes. ${ }^{33}$

Auf den ersten Blick scheint das Ensemble mit der Inschrift in seiner ursprünglichen Anordnung auf uns gekommen zu sein. Die Tatsache, dass die erste Zeile komplett und die zweite Zeile teilweise verdeckt ist, wirft dennoch Fragen auf. Die teilweise verdeckte Inschrift könnte man mit einer Versetzung oder einer späteren Anbringung der Rippen erklären. Der Löwenkopf mit seiner Funktion als Wasserspeier oder Brunnenauslauf ist eindeutig sekundär verbaut. Dies könnte natürlich auch schon zu Bau-

26 Dietl 2009, Bd. 2, 899f.

27 Ebd.; siehe auch Cavallaro 1992, 37.

28 Cavallaro 1989, 333; Cavallaro 1992, 33; Cavallaro 1994, 156.

29 Cavallaro 1989, 331f.; Cavallaro 1992, 31-33; Cavallaro 1994, 154; Dietl 2009, Bd. 2, 899.

30 Cervini 1991, 313-318; Di Fabio 1995, 510 u. 513; Untermann 2001, 222.

31 Viele Autoren halten das Lob in der Inschrift für einen Bauverwalter für recht ungewöhnlich und schließen daher zusammen mit dem Wortlaut auf einen Architekten. Siehe Cavallaro 1989, 332; Cavallaro 1992, 32; Cavallaro 1994, 153f. u. 156; Polonio Felloni 1996, 128f. Anm. 35; Polonio Felloni 1998, 114. Eine Lobschrift, die auf den Scharfsinn der Person abzielt, kann laut diesen Autoren keinem reinen Bauverwalter gewidmet sein. Zudem verweisen sie auf die Bautätigkeiten der Zisterzienser. Andere Autoren folgen dieser Meinung einfach. Siehe Dufour Bozzo 1999, 175; Armillotta 2009/10, 121. Zum architektonischen Bezug des Palastes zur Architektur der Zisterzienser siehe Cavallaro 1992, 22-30; Cavallaro 1994. Clario Di Fabio widerspricht dieser Ansicht zurecht vehement und bezeichnet sie als einen Mythos der lokalen Geschichtsschreibung. Siehe Di Fabio 1995, 510. Matthias Untermann schreibt in Bezug auf den Palazzo di San Giorgio und die Hafenanlage in Genua, dass die dort „genannten Konversen [...] lediglich die administrative und organisatorische Leitung der Bauhütten inne[hatten]. " Siehe Untermann 2001, 222. Allgemein zu der Thematik und Forschungsproblematik von Zisterziensern als Bauleute und Architekten siehe ebd., 208-231.

32 Dietl 2009, Bd. 2, 900.

33 Cavallaro 1992, 33. Siehe auch Dellacasa 1998, Dok. 758: in palacio comunis quod fit in ripa. 
zeiten geschehen sein. Der Inschriftenstein wurde aber angeblich 1262 weiter nach oben versetzt, sodass von der ersten Zeile der Inschrift nur der untere Teil in der Mitte sichtbar war. ${ }^{34}$

Es gibt aber noch eine weitere mögliche Erklärung dafür, dass der Inschriftenstein 1262 nach oben versetzt wurde und der Löwenkopf sekundär eingebaut wurde. Die Inschrift befand sich wohl ursprünglich dort, wo sich heute der Löwenkopf befindet. Die Versetzung der Inschrift dürfte zwei Gründe haben. Am 9. Mai 1262 wurde Capitano Boccanegra wegen Tyrannei abgesetzt. Kurz zuvor war am 5. Mai, laut Genueser Annalen, ein Schiff in Genua eingetroffen, auf dem sich steinerne Trophäen befanden. ${ }^{35}$ Es handelte sich um Beutestücke aus dem Palast der Venezianer in Konstantinopel. ${ }^{36}$ Dieser Palast diente der politischen Repräsentation Venedigs in Konstantinopel. Michael VIII. Palaiologos wollte mit Hilfe der Genuesen Konstantinopel von den Venezianern zurückerobern. Hierzu wurde der Vertrag von Nymphaion (13. März 1261) geschlossen. Bevor aber die Genueser Truppen zur Hilfe kommen konnten, hatte der Kaiser Konstantinopel alleine zurückerobert. ${ }^{37}$ Dennoch durfte Capitano Boccanegra mit Billigung von Michael VIII. Palaiologos, dem byzantinischen Kaiser, den Palast der Venezianer zerstören. ${ }^{38}$ In den Annalen wird auch berichtet, dass die Spolien im Kommunalpalast von Genua als Trophäen verbaut wurden. ${ }^{39}$ Wo sich der venezianische Palast in Konstantinopel befand, konnte bisher nicht geklärt werden. ${ }^{40}$ Über den Transport von Steinen des Venezianer Palastes in Konstantinopel nach Genua berichten die zeitgenössischen Genueser Annalen im Jahr 1262. Die Steine wurden laut den Annalen im Kommunalpalast vermauert. ${ }^{41}$ Mit der domus comunis ist in dem Bericht der Kommunalpalast Genuas gemeint. ${ }^{42}$ Über die Art der Steine berichten die Quellen hingegen nichts. Erst in späteren Quellen werden sie mit „schön“ und „viel“ qualifiziert und quantifiziert..$^{43}$ Rebecca Müller beschreibt die Situation wie folgend:

Der Palast war bereits vollendet, die Bauinschrift direkt über dem Türsturz angebracht. Die Trophäen wurden in das Portaltympanon eingesetzt, wobei eine der Spolien aus Konstantinopel, der Wasserspeier mit den flankierenden Drachen, die Inschrift mit der Nennung des nunmehr

34 Dietl 2009, Bd. 2, 899. Ferrando Cabona beschreibt Arbeitsspuren an den Steinen im Lünettenfeld und schließt daher auf eine spätere Veränderung. Siehe Ferrando Cabona 1998, 42.

35 Müller 2002, 72, 87. Siehe Annales Ianuenses 1926, 45f. Zu den Randzeichnungen und Miniaturen und deren Text-Bild-Bezug in den Annales Ianuenses siehe Haug 2016.

36 Cavallaro 1989, 331; Cavallaro 1992, 31. Ihr folgt Ferrando Cabona 1998, 42.

37 Müller 2002, 72 u. 218.

38 Dietl 2009, Bd. 2, 900.

39 Müller 2002, 72. Siehe Annales Ianuenses 1926, 45.

40 Müller 2002, 72, 217f. Für die Annahme, dass sich der Palast im Bereich des Pantokratorklosters befand, fehlen plausible Begründungen. Siehe Müller 2002, 72, Anm. 142 u. $217 f$.

41 Annales Ianuenses 1926, 45.

42 Siehe hierzu auch Müller 2002, 214.

43 Siehe hierzu Müller 2002, 214. 
zur persona non grata erklärten Capitano ersetzte. Diese verschwand, kaum noch sichtbar, nach oben unter den Portalbogen. ${ }^{44}$

Es wird in der Forschung angenommen, dass fünf Steine aus Konstantinopel im Kommunalpalast verbaut wurden. ${ }^{45}$ Am Bauwerk unterscheiden sie sich vom sonst verwendeten dunklen lokalen Kalkstein. Seit dem 19. Jahrhundert wurden sowohl die Löwenkopfskulptur mit den vier kleinen Tieren als auch die beiden anderen Löwenköpfe, die an der Ostfassade eingebaut sind, damit identifiziert (Abb. 4 und 5). ${ }^{46}$ Die beiden rosafarbenen Steine neben dem Löwenkopf wurden erstmals 1998 von Isabella Ferrando Cabona als Spolien bezeichnet, da dieses Material sonst nicht am Bauwerk vorkommt ${ }^{47}$ Für sie ist der Löwenkopf ein zweitverwendetes Stück, einerseits, da ein Loch im Maul ist und er deshalb wohl zu einem Springbrunnen gehörte, andererseits, da die Ränder wie bei der Inschriftentafel unsauber behauen sind. ${ }^{48}$ Das Artefakt ist somit seiner Funktion entzogen. Der Löwenkopf, der laut Ferrando Cabona aus prokonnesischem Marmor besteht, gehörte zu diesen Trophäen. ${ }^{49}$ Die Analysen des Materials wurden von Tiziano Mannoni und Roberto Ricci durchgeführt und die Herkunft „del mascherone e dei marmi rosa“ als „dell'area del Mediterraneo orientale“ angenommen. ${ }^{50}$

Müller sieht die Analyse als ein gewichtiges Argument dafür, dass das Stück aus Konstantinopel kommt, da in Genua meistens der italienische weiße Marmor verwendet wurde, der üblicherweise in den Marmorbrüchen von Carrara abgebaut wird. Sie sieht daher in dem Relief einen der Steine aus Konstantinopel. ${ }^{51}$ Prokonnesischer Marmor stammt von der Insel Marmara (Prokonnesos) im Marmarameer vor Istanbul. ${ }^{52}$ Zumindest kann man festhalten, dass es sich bei dem Löwenkopf nicht um den italienischen weißen Marmor handelt.

Die von Ferrando Cabona als Spolien bezeichneten rosafarbenen gemaserten Steinquader haben nach den bereits genannten Untersuchungen eine Provenienz „dell'area del Mediterraneo orientale“. Eine genaue Steinsorte nennen sie nicht. Müller vermutet dem Augenschein nach Marmor aus Chios (Portasanta)..$^{53}$ Daher sind sie eventuell auch Beutestücke aus Konstantinopel. Ferrando Cabano nimmt an, dass der Inschriftenstein gemeinsam mit den beiden Marmorblöcken über dem Tür-

\footnotetext{
44 Müller 2002, 87.

45 Ebd., 213-215.

46 Ebd., 214.

47 Ferrando Cabona 1998, 71 Anm. 1.

48 Ebd., 56.

49 Ebd., 56 u. 71 Anm. 1. Albert Dietl übernimmt diese Annahme. Siehe Dietl 2009, Bd. 2, 900.

50 Ebd., 71 Anm. 1.

51 Müller 2002, 215.

52 Zu Marmor von der Insel Marmara (Prokonnesos) aus geologischer Sicht siehe Cramer 2004, 110-123.

53 Müller 2002, 215, Anm. 142.
} 

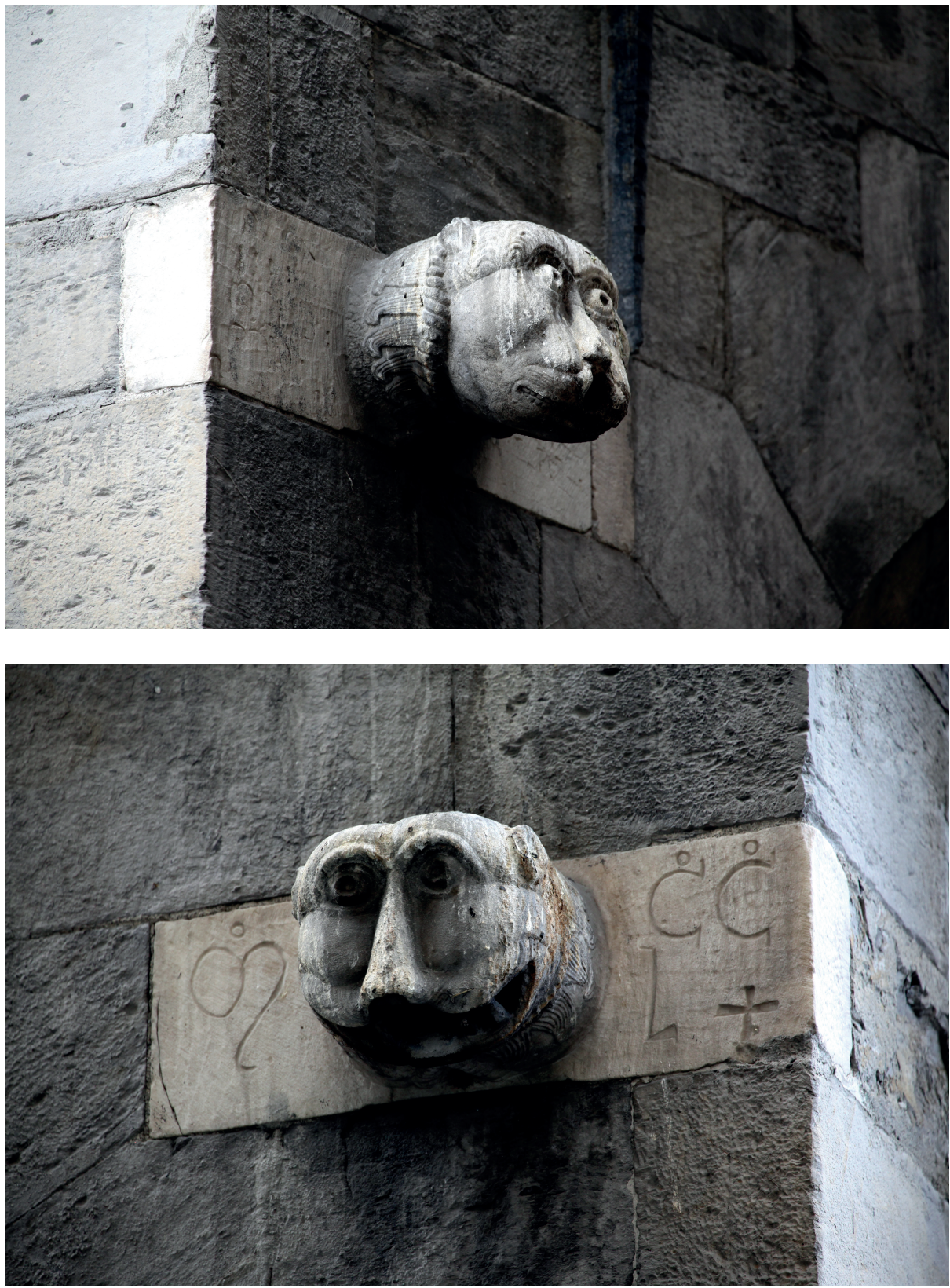

Abb. 4 und 5: Genua, Palazzo di San Giorgio, Ostfassade, Löwenköpfe (Foto @ 
sturz einen Monolithen gebildet hat und somit vor Einbau der Löwenkopfskulptur die Inschrift gut zu lesen war. ${ }^{54}$ Müller weist dies als nicht plausibel zurück, da es hierfür keinen Hinweis gäbe und zudem die gedrängte Schrift und die Anordnung dieser bei einem größeren Stein nicht logisch sei. ${ }^{55}$ Letzteres Gegenargument ist nicht ausreichend, da man genauso gut die Inschrift auch über mehrere Steine hinweg hätte einhauen können. Dies wirft die Frage auf, wieso die Inschrift überhaupt so gedrängt und mit so vielen Zeilen gestaltet wurde. Man hätte sie auch über die ganze Lünettenbreite über mehrere Steine hinweg mit weniger Zeilen einmeißeln und hierdurch eine bessere Sicht- und Lesbarkeit erzielen können. Die Gesamtsituation lässt die Vermutung zu, dass der Inschriftenstein eventuell nicht explizit für diese Stelle gefertigt wurde. Vielleicht wurde einfach ein Stein mit einer Inschrift bestellt oder die Inschrift war ursprünglich für einen anderen Ort am Palast konzipiert worden. Oder sie befand sich sogar an einem anderen Ort und wurde vielleicht erst beim Einbau der Löwenkopfskulptur an diese Stelle versetzt. Oder vielleicht wurden beide sogar erst bei den Umbaumaßnahmen 1570/1571 in das Lünettenfeld vermauert. Auch die gleiche Breite, die der Inschriftenstein und die Löwenkopfskulptur haben, verstärkt diese Frage. Es könnte sich natürlich auch um einen Zufall handeln, da sich z. B. links und rechts des Inschriftensteins weitere Steine mit Inschriften oder auch Skulpturen befanden, die man dann nach dem Einbau zu Gunsten der Löwenkopfskulptur an einer anderen Stelle verbaute.

Die beiden Löwenköpfe, die sich außen an der Ostfassade der Portikus befinden, werden ebenfalls für Trophäen gehalten. ${ }^{56}$ Sie sind an den beiden Fassadenenden jeweils in einer Höhe von ca. 4,5 Metern vermauert und bestehen jeweils aus einem Stück mit den Quadern, die 80 Zentimeter breit und 25 Zentimeter hoch sind. ${ }^{57}$ Das Material ist weißer Marmor, der sonst nicht am Bau verwendet wurde. Da es sich bei beiden Skulpturen wegen der Öffnung im Maul wahrscheinlich ebenfalls um Wasserspeier handelt, sind sie als Spolien zu identifizieren. ${ }^{58}$ Wie Müller bemerkt hat, haben beide vor dem Mähnenansatz einen Strick um den Hals, der an der Unterseite geknotet ist. ${ }^{59}$ Ein Strick würde auf domestizierte Tiere verweisen. Eine Interpretation hierzu steht noch aus. Man könnte dies als einen Hinweis auf das von den Genuesen besiegte und gebändigte Venedig deuten.

54 Ferrando Cabona 1998, 44.

55 Müller 2002, 87.

56 Die Begründung, dass dies allein wegen des Symbols der Republik Venedig der Fall sein müsste, hat bereits Müller als nicht überzeugend zurückgewiesen, da die Löwen nicht das charakteristische Aussehen des Markuslöwen haben und dieser erstmals für das Jahr 1262 als Symbol für den Staat Venedig nachgewiesen ist. Siehe Müller 2005, 107 Anm. 21. Zur Begründung siehe Weigel 1996, $127 f$.

57 Müller 2002, 215.

58 Ebd., 216.

59 Ebd., 215. 
Am nördlichen Löwenkopf sind in gotischer Majuskel Zahlen eingemeißelt. Links des Kopfes das M, rechts das CC und darunter $\mathrm{L}+.^{60}$ Dies ergibt die Jahreszahl des Baubeginns 1260. Diese Jahreszahl ist eventuell erst zur Zweitverwendung eingemeißelt worden. Die Löwenköpfe scheinen von östlicher Kunst beeinflusst zu sein. Allgemein wurden Löwenköpfe in Byzanz schon früher als Wasserspeier und als Brunnenausflüsse verwendet als im Westen. ${ }^{61}$ Der Löwenkopf am Portal wurde später eingesetzt, was durch die Erhöhung der Inschriftentafel deutlich wird. Er kann also vor allem wegen des zeitlichen Zusammenhangs zwischen der Ankunft der Spolien und der Erhöhung der Inschriftentafel aus der Beute vom venezianischen Palast in Konstantinopel stammen. ${ }^{62}$ Löwenköpfe als Wasserspeier sind bereits in der Antike bekannt. Müller verweist auf Löwen-Aquamanile in Hinsicht auf die Funktion als Wasserspeier und auf die Ikonographie. ${ }^{63}$ Sie schreibt über die Herkunft der Löwenkopfskulptur: „Der Versuch einer stilistischen Einordnung des Wasserspeiers wurde bislang nicht unternommen. Er erweist sich aufgrund der insgesamt groben Ausführung als problematisch und erlaubt keine Aussage über die Provenienz."64 Philipp Niewöhner vergleicht die Skulpturen mit Stücken aus Byzanz, um eine dortige Provenienz $\mathrm{zu}$ belegen. Als Vergleiche dienen ihm ein Löwenaquamanile und eine Schale, die deutlich jünger ist. ${ }^{65}$ Zuletzt verweist er auf Löwenköpfe an den mittelbyzantinischen Kapitellen im Narthex von San Marco in Venedig und auf einen mittelbyzantinischen Kämpferbalken mit Löwenköpfen vom Topkapı-Tor in Istanbul. ${ }^{66}$

Robert S. Nelson hat die beiden Löwenköpfe an der Ostfassade des Genueser Kommunalpalastes mit denen an den Bronzetüren von San Marco in Venedig verglichen, ${ }^{67}$ die aus Byzanz importiert wurden. ${ }^{68}$ Löwenköpfe an Bronzetüren waren in mittelbyzantinischer Zeit sehr weit verbreitet und wurden zum Halten des Türringes benutzt. ${ }^{69}$

60 Zur Inschrift siehe Remondini 1874, 94. Bereits Carlo Cuneo hat die Jahreszahl im Gegensatz zu späteren Autoren richtig erkannt und publiziert. Siehe Cuneo 1842, 197. Frederico Alizeri erkannte in der Jahreszahl das Jahr 1250, da er das X nicht als Zehn las. Siehe Alizeri 1847, Bd. 2, 274. Die Inschrift fehlt bei Silva 1987. Ferrando Cabona schreibt unverständlicherweise immer noch 1250. Siehe Ferrando Cabona 1998, 56.

61 Siehe hierzu: Niewöhner 2013/2014; Niewöhner 2016.

62 Niewöhner 2013/2014, 84; Niewöhner 2016, 172.

63 Müller 2002, 215.

64 Ebd. Inzwischen gibt es einen Versuch der Einordnung in die byzantinische Kunst, auf die später eingegangen wird.

65 Niewöhner 2013/2014, 85f. u. Abb. 20 und 21; Niewöhner 2016, 175.

66 Niewöhner 2013/2014, 85 u. Abb. 23, 24; Niewöhner 2016, 176 u. Abb. 9.12, 9.13.

67 Nelson 2010, 76 Anm. 43. Zu den Bronzetüren siehe: Iacobini 1997, hier auch Abb. 11-31. Bereits Müller führte die Löwenköpfe an den Bronzetüren in Venedig als Vergleich auf. Siehe Müller 2002, 216.

68 Niewöhner 2013/2014, 85. Siehe hierzu auch Vio 2009 und Paribeni 2009. Ursula Mende vermutet eine Entstehung der Löwenköpfe an den Bronzetüren in Venedig selbst. Siehe Mende 1981, 231 Kat. 58, hier auch Abb. 105-111.

69 Niewöhner 2013/2014, 85. 
Die beiden Skulpturen in Genua können daher nach Niewöhner vom venezianischen Palast in Konstantinopel stammen und auf die Tradition der byzantinischen Wasserspeier mit Löwenköpfen zurückgehen. Es können daher in Byzanz gearbeitete Stücke sein, wenn der Palast älter und damit byzantinisch war oder wenn die Venezianer byzantinische Spolien verwendet oder byzantinische Bildhauer angestellt haben. ${ }^{70}$ Vom Stil her gleichen die Köpfe laut Niewöhner vor allem in den Augenpartien und in der Gestaltung des Schnurrbarts einem mittelbyzantinischen Löwenkopf, der beim Topkapı Sarayı ausgegraben wurde und sich heute im Archäologischen Museum von Istanbul befindet. Der Kopf soll aus Prokonnesischem Marmor sein. ${ }^{71}$ Für Niewöhner sind die von ihm genannten Argumente und das Fehlen sowohl von figürlichen Wasserspeiern als auch von stilistischen Vergleichen in dieser Zeit im Westen ein Beleg dafür, dass die drei Stücke vom venezianischen Palast aus Konstantinopel, der für ihn wahrscheinlich ein mittelbyzantinischer Palast war, kamen. ${ }^{72}$ Die Argumentation scheint zunächst schlüssig. Vor allem das Argument mit den fehlenden figürlichen Wasserspeiern ist ausschlaggebend. Allerdings sind fehlende stilistische Vergleiche auch im Westen kein Argument, wenn man motivische Vergleiche aus Byzanz vorzieht. Die Vergleiche von Niewöhner sind genauso entfernt wie die von Müller selbst als entfernte Vergleiche eingestuften westlichen Stücke. ${ }^{73}$ Motivische Vergleiche lassen sich nämlich durchaus aus dem Westen finden. Zudem sollte man bedenken, dass das Formengut zu dieser Zeit allgegenwärtig war. Man findet so zeitlich vorher im Westen Löwenköpfe an Bronzetüren wie z. B. in Mainz oder Löwenaquamanile wie z. B. aus Hildesheim oder auch Löwenköpfe an Basen wie im Wormser Dom oder an Kapitellen wie in San Michele in Pavia. ${ }^{74}$

Es lässt sich also bisher weder stilistisch noch motivisch belegen, wo die Stücke hergestellt worden sein könnten. Von den Motiven und dem Stil her könnten die Skulpturen ohne Probleme auch in Oberitalien hergestellt worden sein. Bereits Robert S. Nelson schrieb bei seinem Vergleich der Löwenköpfe von der Ostfassade mit denen der Bronzetüren in Venedig: „The comparison suggests that the heads now at Palazzo di San Giorgio were carved by a Venetian artist in Constantinople from local marble. “75

70 Ebd.; Niewöhner 2016, $173 f$.

71 Niewöhner 2013/2014, 85 u. Abb. 19; Niewöhner 2016, 174.

72 Niewöhner 2013/2014, 87. In der neueren Fassung des Artikels finden die Argumente des Fehlens von figürlichen Wasserspeiern in dieser Zeit im Westen und die fehlenden stilistischen Vergleiche im Westen keine Erwähnung mehr. Siehe Niewöhner 2016.

73 Müller 2002, 216f.

74 Eine genauere Einordnung kann in dieser Studie nicht vorgenommen werden. Dies ist an anderer Stelle zu leisten.

75 Nelson 2010, 76 Anm. 43. Niewöhner, der in der ersten Fassung seines Artikels den Vergleich von Nelson an sich erwähnt, berücksichtigt diese Aussage nicht, da sie nicht in seine Argumentation passt. Siehe Niewöhner 2013/2014, 85. 
Die Marmorart der beiden Löwenköpfe an der Ostfassade ist bisher noch nicht chemisch untersucht worden. Aber laut mikroskopischer Analyse von Tiziano Mannoni scheint es sich am ehesten um Marmor aus Carrara zu handeln. ${ }^{76}$ Dies deutet nicht auf eine Herkunft aus Konstantinopel hin.

Ein Blick auf die Ausarbeitung unterstützt diese These. Bereits Müller war der Meinung, dass die Stücke stilistisch nicht mit dem anderen Löwenkopf zusammenpassen, ${ }^{77}$ hatte eine Einordnung im oberitalienischen Raum versucht und laut eigener Aussage nur entfernte Vergleiche gefunden. ${ }^{78}$ Eine Herkunft aus Konstantinopel erscheint daher eher unwahrscheinlich. Meiner Meinung nach ist die Art der Lippenhaargestaltung für oberitalienische Bauskulptur im 12. und 13. Jahrhundert typisch. Es ist also durchaus denkbar, dass die beiden Löwenköpfe an der Ostfassade in Italien durch oberitalienische Bildhauer geschaffen wurden. ${ }^{79}$

Es sind technologische Untersuchungen erforderlich, um festzustellen, ob es sich bei den beiden Löwen an der Fassade um Marmor aus Prokonnesos oder aus Carrara handelt. ${ }^{80}$ Es besteht allerdings auch die Möglichkeit, dass die Skulpturen von oberitalienischen Bildhauern in Konstantinopel geschaffen wurden, oder dass die Stücke in Genua von der Kommune sogar als falsche Trophäen in Auftrag gegeben wurden. Eventuell wurden sie aber auch gar nicht als Trophäen angesehen.

Die Inschrift beim Löwenkopf am Portal wurde höchstwahrscheinlich nach oben versetzt. In der Forschung wird immer wieder behauptet, dass hierdurch die erste Zeile mit dem Namen des geächteten Capitano verdeckt werden sollte. Hierdurch sei der Name hinter dem Bogenscheitel in einem Akt der damnatio memoriae verschwunden. ${ }^{81}$ Niewöhner sieht hierin eine Möglichkeit, die Aufmerksamkeit vom Namen des Capitano abzulenken, ohne die Inschrift abzunehmen und damit eine potentiell politisch gefährliche Aussage zu machen. ${ }^{82}$ Die Trophäe sei für das Prestige wichtiger gewesen als die Erinnerung an den eben verbannten Capitano. ${ }^{83}$

Die Sichtbarkeit ist allerdings anders als sie bisher in der Forschung wiedergegeben wurde: Der Name ist am Ende der zweiten und am Anfang der dritten Zeile wie-

76 Müller 2002, 216.

77 Ebd.

78 Ebd., $216 \mathrm{f}$.

79 Eine stilistische Einordnung kann im Rahmen dieser Studie nicht geleistet werden.

80 Manche Marmorarten lassen sich nicht mikroskopisch unterscheiden. Vor allem die isotopischen Merkmale sind bei den Untersuchungen von großer Bedeutung. So wurde erst um die Jahrtausendwende festgestellt, dass bei prokonnesischem Marmor entgegen der bisherigen Annahmen ein zweites Isotopenfeld besteht. Hierdurch lassen sich bei Analysen die ähnlich aussehenden Marmorarten unterscheiden und man kann nun feststellen, ob ein Marmorstück aus prokennesischen Marmor besteht oder nicht. Siehe Cramer 2004, 120-122.

81 Müller 2002, 87.

82 Siehe hierzu Niewöhner 2013/2014, 84f.; Niewöhner 2016, 173.

83 Cavallaro 1992, 31. 
dergegeben. Hierdurch ist nur der Nachnahme am Ende der zweiten Zeile teilweise durch die Rippe verdeckt bzw. häufig verschattet. Zudem erschwert der Löwenkopf eine Sicht auf den Namen, wenn man vor dem Portal steht und es durchschreitet. Wieso wurde also das Lesen der Inschrift nur etwas erschwert? Wieso hat man die Inschrift nicht einfach komplett entfernt? Dies dürfte mehrere Gründe gehabt haben: Einmal spricht das Gebäude selbst zu uns, dass es 1260 erbaut wurde. Die Inschrift gehört also zum Gebäude. Zudem wird mit Frater Oliverius auch der Bauverwalter genannt, den man weiterhin ehren wollte bzw. dessen memoria man nicht verhindern wollte. Die Versetzung lässt sich also darauf zurückführen, dass der Auftraggeber des Palastes wenige Zeit später in Ungnade gefallen war. Die Skulptur dient hierbei der Verschleierung der Inschrift. Zudem wollte man die Trophäe an einer prägnanten Stelle anbringen. Eine Trophäe mit der Anbringung von städtischen Hoheitszeichen, hier dem Wappen von Genua, repräsentiert nach Müller eine wehrhafte Kommune. ${ }^{84}$ Dem Genueser Bürger sollen am Portal auf dem Weg zum Hafen durch die Trophäe die Siege der kommunalen Seemacht Genua präsentiert werden. Die Trophäe ist also aus mehreren Gründen bewusst an dieser Stelle zusammen mit der Inschrift und dem Stadtwappen der Kommune als Zeichen der Macht prägnant platziert worden. Die Skulptur verdeckt teilweise die Inschrift und erschwert dadurch das Lesen des Namens des in Ungnade gefallenen Auftraggebers. Für den früheren Podestà Guglielmo Boccanegra ist es natürlich tragisch, da dies genau mit einer Trophäe erfolgte, die er selbst erbeutet hatte. Der Betrachter wird aber zumindest teilweise an die Ikonographie der Löwenskulptur gedacht haben. Er wird sowohl an die Wächterfunktion des Löwen als auch an den Löwen als wehrhaftes Tier und als König der Tiere, der daher auch immer ein Repräsentant von Herrschaft und Macht ist, erinnert worden sein. ${ }^{85}$ In den kleinen Löwen könnten die Kinder der Stadt, die Bürger, gesehen werden, die einmal vom großen Löwen, der Kommune, geschützt werden oder diesen im Kampf unterstützen. Sie sind aggressiver dargestellt, da sie die Zähne blecken. Ihnen fehlt aber als Individuum die Macht, die die Kommune als Gesamtheit hat. Die Drachen könnte man als Feinde interpretieren, die den Löwen und damit die Kommune angreifen wollen. Diese können von außen oder eben auch von innen stammen, wie dies beim früheren Podestà der Fall war. Die Skulptur kann also in ihrer neuen Funktion als Trophäe am Portal im Zusammenhang mit dem Kommunalpalast und der Inschrift als Zeichen der kommunalen Macht interpretiert werden.

84 Müller 2002, 88.

85 Zum Löwen als Zeichen der Herrscher im Mittelalter siehe Jäckel 2006. 


\section{Das Reiterstandbild des Oldrado da Tresseno am Broletto in Mailand}

In Mailand befindet sich am Palazzo della Ragione, dem Broletto, dem mittelalterlichen Ratsgebäude, an der Südfassade ein Reiterstandbild des Oldrado da Tresseno (Abb. 6 und 7). ${ }^{86}$ Der Palazzo war in der Mitte des Hofplatzes und teilte diesen in zwei Hälften. Der Platz wurde von einer Mauer mit sechs Toren umgeben, von denen aus Straßen zu den sechs Stadttoren führten. Durch dieses Straßennetz war der Platz eindeutig als das Zentrum der Kommune markiert. Der Palazzo ersetzte das alte kommunale Verwaltungszentrum, das sich im Bereich des erzbischöflichen Palastes neben dem Dom Santa Maria Maggiore befand. ${ }^{87}$ Unter dem Podestà Aliprando Fava da Brescia hatte 1228 die Kommune beschlossen, ein neues Stadtzentrum zu bauen. ${ }^{88}$ Bereits für das Jahr 1229 ist eine erste Benutzung überliefert. ${ }^{89}$ Der Bau muss also schnell vorangeschritten sein, sodass er in Teilen benutzt werden konnte. Der Palazzo ist ein longitudinaler Bau. Das Untergeschoss besteht aus einer offenen Pfeilerhalle. Auf den Längsseiten ist sie durch sieben und auf den Schmalseiten durch zwei Arkaden gegliedert. Darüber befindet sich ein Saalgeschoss aus Ziegelmauerwerk, das durch dreiteilige Fenster, die jeweils über den Arkaden liegen, belichtet wird. Am 7. Januar 1771 wurde beschlossen, dass der Palazzo della Ragione der Sitz des notariellen Archivs werden sollte. ${ }^{90}$ Daraufhin beauftragte man Francesco Croce den Bau zu renovieren und um ein Geschoss aufzustocken. ${ }^{91}$ Hierbei wurde der Bereich mit dem Reiterstandbild nicht beeinträchtigt.

Das Reiterstandbild ist in einer Blendnische oberhalb der Arkadenzone an der Nordseite angebracht. Die Nischenarchitektur, die sowohl aus Marmor als auch aus Kalkstein und noch weiteren Gesteinsarten besteht, beginnt von Osten noch im Arkadenzwickel zwischen der dritten und vierten Arkade. Das etwa 140 Zentimeter hohe und 40 Zentimeter breite Reiterstandbild besteht zusammen mit dem Rahmen und der Standplatte aus einem Marmorblock. ${ }^{92}$

Ein jung wirkender Reiter sitzt aufrecht in einem schlichten und leichten Gewand und Mantel gekleidet auf seinem Pferd. Seinen Kopf hat er leicht nach vorne gebeugt,

86 Zum Palazzo della Ragione siehe: Paul 1963, 49-51, 149; Grimoldi 1983; Bering 1986, 147-159; Bocchi 1993.

87 Paul 1963, 148; Seiler 1989, 69f.

88 Grimoldi 1983, 72; Seiler 1989, 70. Siehe auch Lomartire 2015, 104 Anm. 27.

89 Grimoldi 1983, 72 u. 73 Anm. 8.

90 Ebd., 87.

91 Ebd., 96f. In der zweiten Hälfte des 19. Jahrhunderts und zu Beginn des 20. Jahrhunderts bestand das Vorhaben, das Obergeschoss wieder rückzubauen. Siehe hierzu ebd., 122-145. Zu Restaurierungen siehe ebd., 36-57.

92 Lomartire 2015, 110. Zur Breite siehe auch: Grimoldi 1983, 149. Zu den gefundenen Fassungsresten siehe Lormatire 2015, $110 f$. 

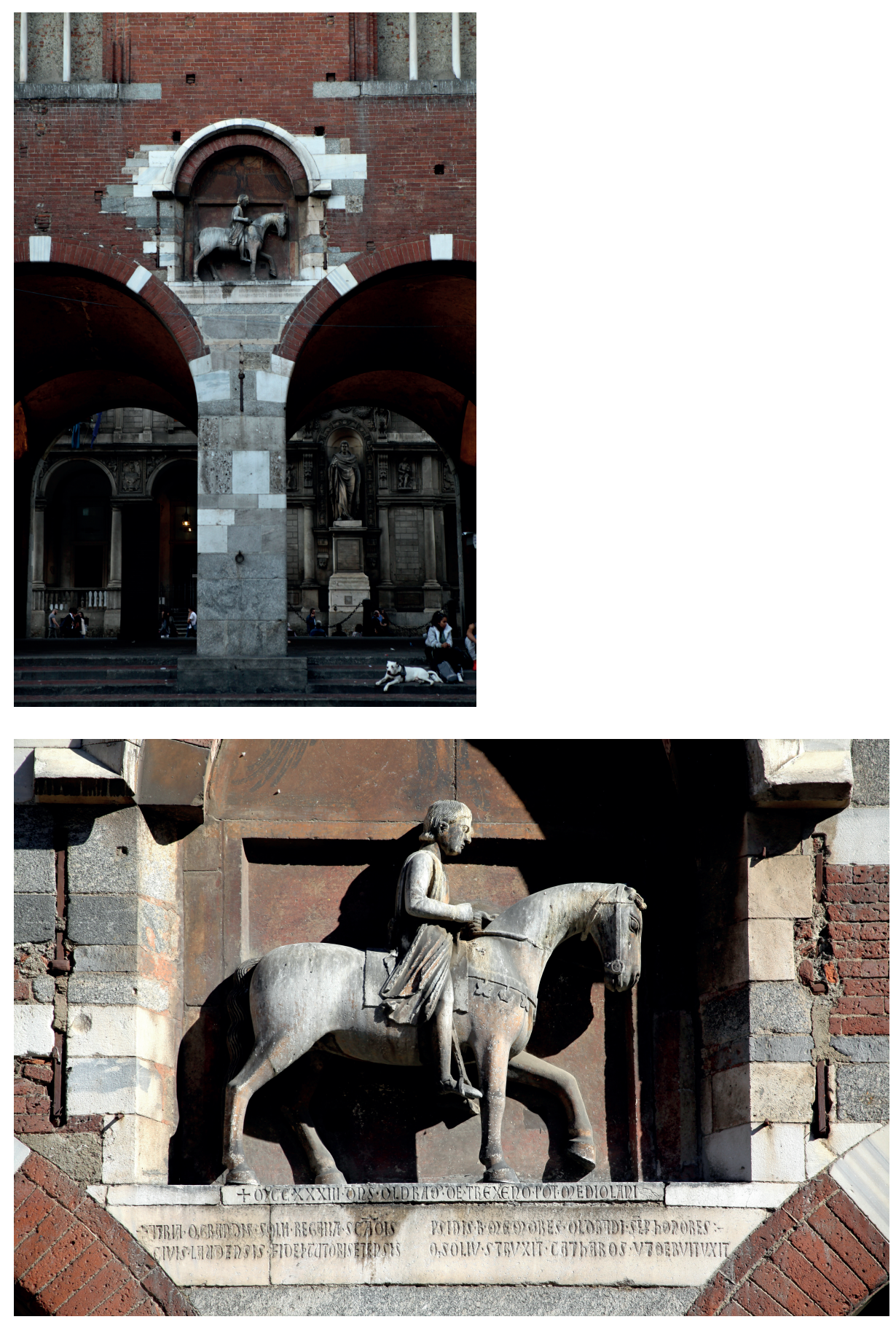

Abb. 6 und 7: Mailand, Broletto, Südfassade, Reiterstandbild des Oldrado da Tresseno (Foto $\odot$ Wilfried E. Keil). 


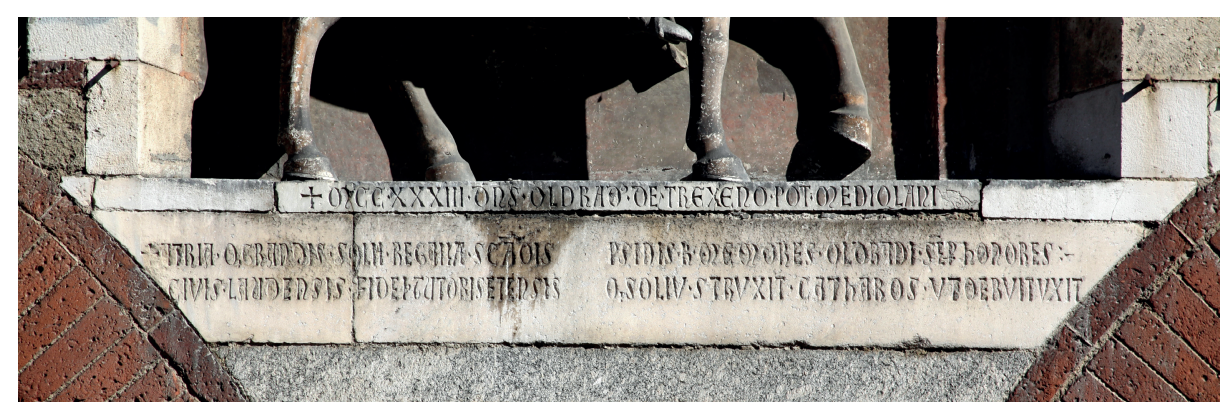

Abb. 8: Mailand, Broletto, Südfassade, Reiterstandbild des Oldrado da Tresseno, Inschrift (Foto () Wilfried E. Keil).

um sich zielstrebig auf seinen Weg und damit auf das vor ihm Liegende mit Ausdauer zu konzentrieren. Mit gestreckten Beinen steckt er mit den Füßen in den Steigbügeln; seine Zehen zeigen zum Boden. Der Reiter hält die Zügel recht kurz, um das Pferd im gemäßigten Schritt zu halten. Seine Arme hat er angewinkelt. Mit der linken Hand hält er sich am Sattelknauf fest und mit der rechten Hand scheint er früher etwas gehalten zu haben. Über dem Reiter sieht man im Blendbogenfeld noch einen gemalten Adler, der die kaiserliche Herrschaft symbolisiert. ${ }^{93}$

Auf der Vorderseite der Standplatte des Reiters ist eine einzeilige Inschrift in gleicher Länge eingemeißelt. Auf den beiden Steinen darunter steht in zwei Zeilen eine längere Inschrift mit größeren Buchstaben. Beide Inschriften erscheinen eher als Beiwerk, was zudem auch an einer heute fehlenden Farbfassung liegt. Die Inschrift auf der Standplatte in gotischer Majuskel lautet (Abb. 8):

+ MCC $\cdot$ XXXIII $\cdot$ D(OMI)N(V)S $\cdot$ OLDRAD(VS) $\cdot$ DE TREXENO $\cdot$ POT(ESTAS) $\cdot$ MEDIOLANI

1233 Herr Oldradus von Dresano, Podestà von Mailand.

Im Anschluss bildet ein kleiner Drache das Schlusszeichen der Inschrift. Die Inschrift nennt also das Jahr der Entstehung bzw. Fertigstellung des Gebäudes, den Namen des Reiters und seine Funktion. Bei Trexeno handelt es sich wahrscheinlich um einen Ort namens Dresano, der südwestlich von Mailand nahe Melegnano liegt. ${ }^{94}$ Die darunter liegende Inschrift, ebenso in gotischer Majuskel, besteht aus zweimal zwei Zeilen. Es handelt sich um zwei Distichen in leoninischen Hexametern.

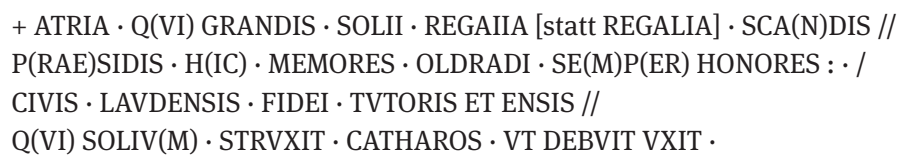

93 In der Forschung wird der Adler als kaiserlicher Adler gedeutet. Siehe Seiler 1989, 68. 94 Lomartire 2015, 103. 
Der du die Atrien, den Gerichtssitz besteigst, gedenke des Oldrado, Bürger aus Lodi, Beschützer des Glaubens und des Schwertes, ehre immer den, der den Palast erbaute und der, wie es sich gehörte, die Katharer verbrannte. ${ }^{95}$

Beide Inschriften sind nach paläographischen und metrischen Untersuchungen von Saverio Lomartire zur gleichen Zeit entstanden. ${ }^{96}$

Durch die Inschriften wird nicht nur der Auftraggeber und das Jahr 1233 benannt. Die Inschrift spricht den Vorbeigehenden direkt an und weist darauf hin, dass jeder, der durch die Arkaden schreitet und das Innere des Palastes betritt, sich an die Taten des Podestà erinnern soll. Und dann werden zudem diejenigen explizit angesprochen, die den Gerichtssitz besteigen, also die Nachfolger im Amt des Podestà sind. ${ }^{97}$ Als Taten werden die Erbauung des Palastes und die Vernichtung der Katharer, Anhänger einer häretischen Glaubensrichtung, genannt. Dies zielt auf die memoria des Dargestellten ab. ${ }^{98}$ Zur Ketzerverfolgung wurde in Mailand erst 1228 eine Verordnung erlassen. Die Strafe wurde zunächst nicht angewandt. Erst unter Oldrado da Tresseno wurden in Mailand Ketzer auf dem Scheiterhaufen verbrannt. ${ }^{99}$

Hier stellt sich die Frage der Rezeption der Inschrift. Wurde diese bewusst wahrgenommen oder war eher das Standbild entscheidend?

Der Palast wurde zwischen 1228 und 1233 erbaut. Dies waren zudem auch die Regierungsdaten von Oldrado. In den Mailänder Chroniken des 12. Jahrhunderts wird die Skulptur nicht erwähnt. ${ }^{100}$ Erst im 14. Jahrhundert wird das Reiterstandbild zweimal erwähnt. Aus den Mailänder Annalen geht hervor, dass das Gebäude 1233 fertiggestellt und das Reiterstandbild aufgestellt wurde. ${ }^{101}$

Galvano Fiamma bezeichnet die Anbringung des Standbildes als großen Fehler. ${ }^{102}$ Früher ging man davon aus, dass 1233 auch das Todesjahr von Oldrado war. Lomartire

95 Die Inschrift wurde erstmals 1503 transkribiert. Siehe Corio 1554, 96.

96 Lomartire 2015, 101 Anm. 6.

97 Andrea von Hülsen-Esch ist der Meinung, dass die Inschrift nur an die Nachfolger Oldrados gerichtet sei. Dies basiert auf ihrer Übersetzung des Anfangs: „Wer auch immer du bist, der du den Thron (Gerichtssitz) besteigst, ..." Siehe von Hülsen-Esch 1994, 24. Hierbei wird nicht berücksichtigt, dass zuerst die Personen angesprochen werden, die den Innenhof betreten, und dann erst die Nachfolger im Amt.

98 Zur kommunalen Erinnerungskultur im italienischen Stadtraum im Allgemeinen siehe von der Höh 2006, 21-38.

99 Seiler 1989, 71. Dies geht aus diversen Belegen hervor wie z. B. Galvaneus de la Fiamma 1727, 672. Bei Seiler 1989, 71f. Anm. 311 weitere Belege. Genaueres zur Ketzerverfolgung in Mailand zu dieser Zeit siehe bei Seiler 1989, 71-73.

100 Seiler 1989, 69.

101 Annales Mediolanensis 1730, 643: Isto anno finitum fuit palatium, quod est in medio broleti, ubi in marmore fuit sculptus ipse potestas super equum insidens.

102 Galvaneus de la Fiamma 1727, 672. In seinem Werk Manipulus Florum schreibt er: Tunc alatium broleti novi erigitur, in cuius latere ipse potestas in marmore super equum residens sculptus fuit: quod fuit magnum vituperium. Peter Seiler erörtert die Argumente für diese Bezeichnung. Es könnte sich 
hat in den Genueser Annalen einen „Oldradus Grossus de Trexeno Laudensis“ gefunden, der 1237 Podestà in Genua war und kann ihn im Gegensatz zu einem anderen Oldrado mit dem Oldrado da Tresseno in Mailand gleichsetzten. ${ }^{103}$ Dies macht deutlich, dass die bisherige Annahme des Todesjahres 1233 nicht stimmt.

Die Skulptur des Oldrado da Tresseno ist das älteste erhaltene profane nachantike Reiterstandbild in Italien. ${ }^{104}$ Das Reiterstandbild orientiert sich an antiken Reiterstandbildern. Diese waren im Mittelalter durchaus noch bekannt. In Rom stand das Reiterstandbild des Marc Aurel, der als Konstantin interpretiert wurde, am Lateransplatz. ${ }^{105}$ In der Nähe von Mailand stand in Pavia das antike bronzene Reiterstandbild des Regisole bis ins 18. Jahrhundert auf dem Domplatz. Man hielt den Dargestellten für Theoderich und holte zur Zeit Karls des Großen das Standbild von Ravenna nach Pavia. ${ }^{106}$ Die beiden antiken Reiterstatuen nahmen neben ihrer ursprünglichen Bedeutung als Herrschaftszeichen seit dem frühen Mittelalter auch „als Rechtssymbole eine wesentliche Funktion im öffentlichen Leben“ ein. ${ }^{107}$ Reiterstandbilder wurden immer in Verbindung mit einem Gerichtsort gesehen. Daher sind sie als Rechtssymbole aufzufassen. $^{108}$

Auch Kaiser im Mittelalter, wie Friedrich II., ließen sich bekanntlich als Reiter darstellen, so z. B. bei den drei Portalen von Castel del Monte. ${ }^{109}$ Die Darstellung eines Podestà in einer monarchischen Tradition kann man deswegen auch als Provokation verstehen. ${ }^{110}$ Ein Reiterstandbild war ein Symbol kaiserlicher Macht und im Falle eines Podestà ein Symbol der Macht der Kommune. Die Kommune und ihr Podestà erhoben hiermit einen Herrschaftsanspruch auf die gleiche Stufe wie ein Kaiser. Dies wird nur durch das Bildwerk selbst ausgesagt. Diese Machtdemonstration wird nur durch die Sehgewohnheiten der Rezipienten und nicht durch den Inhalt der Inschrift wirksam.

darum handeln, dass Oldrado nicht der alleinige Erbauer war, oder Galvaneus de la Fiamma (12831344?) betrachtete die Verdienste um die Ketzerverfolgung als negativ. Siehe hierzu: Seiler 1989, $75 f$. Jedenfalls war diese Meinung damals nicht stark genug, um eine damnatio memoriae zu erreichen, wie dies bei anderen Fällen in späterer Zeit geschah. Siehe Lomartire 2015, 105.

103 Ebd., 104. Oldradus Grosso wird in zwei Dokumenten von 1220 und 1221 Konsul von Lodi genannt. Zudem findet sich sein Name zusammen mit einer Gruppe von Antiimperialen. Siehe Lomartire 2015, 103.

104 Valentiner 1956, 135f.; Liebenwein 1983, 119f.; Seiler 1989, 68.

105 Zum Reiterstandbild von Marc Aurel und zu seiner Rezeption im Mittelalter siehe Gramaccini 1985. 106 Grandi 1988, 240; Lomartire 2015, 120f. Zum Regisole in Pavia siehe auch Saletti 1997; Lomartire 2008. Das Aussehen des Reiterstandbildes ist durch eine Federzeichnung mit der Ansicht der Kathedrale von Pavia aus den Jahren 1335-1336 überliefert. Siehe Opicino de Canistris, Liber de laudibus Civitatis Papiae, Biblioteca Apostolica Vaticana, cod. Pal. Lat. 1993, fol. 2 v. Zu einer Abb. siehe Lomartire 2015, 121.

107 von Hülsen-Esch 1994, 25.

108 Ebd., 26.

109 Siehe z. B.: van Cleve 1972, 345.

110 Siehe auch Lomartire 2015, 106. 
Aus der Chronik des Salimbene de Adam, eines Franziskanermönchs aus Parma aus den 80er Jahren des 13. Jahrhunderts, wissen wir, dass es kein Einzelfall war, ein Reiterstandbild für einen Podestà aufzustellen. Bereits 1229 wurde Nazaro Ghirardini da Lucca, dem Podestà von Reggio, ein Reiterstandbild über dem Stadttor, der Porta di Bernone, errichtet. Er hatte Teile der Stadtmauer und auch dieses Tor erbauen lassen. ${ }^{111}$ Das Standbild stand dort bis zum 16. Jahrhundert und danach wurde es wohl zerstört. Von diesem Reiterstandbild befindet sich heute ein Fragment in den Musei Civici in Reggio Emilia. ${ }^{112}$

Das Reiterstandbild des Oldrado da Tresseno wird immer wieder Benedetto Antelami zugeschrieben. ${ }^{113}$ Es würde sich dann allerdings um ein extremes Spätwerk handeln ${ }^{114}$ oder es handelt sich, wie andere meinen, um ein Werk eines Meisters aus seiner Werkstatt. Stilistisch orientiert sich das Standbild an den Reiterskulpturen des Benedetto Antelami. ${ }^{115}$ Dies sind zum einen der Heilige Georg und der Fall des Paulus am Bischofsthron im Dom zu Parma ${ }^{116}$ und die Monatsdarstellung Mai im Baptisterium in Parma. ${ }^{117}$ Am dritten Obergeschoss des Glockenturms des Grossmünster in Zürich befindet sich eine Reiterfigur aus drei Blöcken von lokalem granitischem Sandstein, die sich an den Werken Antelamis orientiert und die dem Reiterstandbild des Oldrado sehr ähnelt. Wen der Reiter in Zürich darstellt, ist ungewiss. ${ }^{118}$ Diese Reiterstandbilder zeigen zudem, dass den Betrachtern Reiter auch aus anderen Kontexten bekannt waren.

Was für einen Gegenstand könnte Oldrado in der Hand gehalten haben? Peter Seiler vermutet hier eine Amtsinsignie wie einen Stab oder ein Schwert. ${ }^{119}$ Lomartire hält ein Schwert für am wahrscheinlichsten. Er ist der Meinung, dass sich das Teil, das unter der geschlossenen Hand erhalten hat, ein Schwertknauf sein könnte. ${ }^{120}$ Ein Schwert steht als Symbol für das Richtschwert und damit für die Rechtsgewalt der Kommune. ${ }^{121}$ Dies lässt sich auch durch die Inschrift belegen. In dieser wird der Podestà auch als Beschützer des Schwertes bezeichnet. Die Gerichtsbarkeit wird also in beiden Ebenen, Schrift und Bild, aufgezeigt und dadurch dem Rezipienten deut-

111 Salimbene de Adam 1998, Bd. 1, 98. Siehe auch Grandi 1988, 240; Lomartire 2015, 105.

112 Marchesini 2008, 558.

113 Diese Zuschreibung wurde erstmals von Adolfo Venturi vorgenommen. Siehe Venturi 1904, 340.

114 Arturo Calzona hat bisher als Letzter das Reiterstandbild als ein Spätwerk des Antelami bezeichnet. Siehe Calzona 2008, 110-115, bes. 110.

115 Grandi 1988, 244, 247; Seiler 1989, 68; Toesca 1927, 892 Anm. 23; Lomartire 2015, 114-118.

116 Woelk 1995, 205-210 u. Abb. 103, 104. Zu den Abb. siehe auch: Calzona 2008, 24 f.

117 Frugoni 1995, 130f. u. Abb. 87. Zu Abb. siehe auch Woelk 1995, Abb. 130, 132; Calzona 2008, 99.

118 Reinle 1969 u. Abb. 4-10; Gutscher 1983, 132f. u. Abb. 162; Claussen 1994, 568f. u. Abb. 10. Adolf Reinle sieht ihn als ein namenloses Symbol der kaiserlichen Pfalz oder als Dietrich von Bern, also König Theoderich. Siehe Reinle 1969, $43 f$.

119 Siehe Seiler 1989, 68.

120 Lomartire 2015, 128.

121 Ebd. 
lich bewusst gemacht. Der Reiter hielt das Schwert nach der Arm- und Handhaltung symbolisch wie ein Herrscher und nicht wie ein Krieger zum Angriff. Der Unterschied zur üblichen Herrschergestalt zeigt sich in der vorwärts geneigten, zielstrebigen Kopfhaltung, die die Dynamik der Kommune versinnbildlichen soll. Die Inschrift dient nicht nur der Benennung des Dargestellten, sondern verdeutlicht auch das Dargestellte. Sie dient also eher dem Bildnis als das Bildnis einer Veranschaulichung des Textes. Durch die Inschrift wird der Betrachter zum Gedenken des Dargestellten und seiner Taten aufgerufen und er erfährt hierdurch zusätzliche Informationen. Der gemalte Adler in der Nischenarchitektur verweist auf Herrschaft. Allerdings ist er auch ein kaiserliches Wappentier. Manchmal wurde der kaiserliche Adler dahingehend interpretiert, dass Oldrado ein „Vollstrecker des kaiserlichen bzw. päpstlichen Willens“122 gewesen sei. Seiler hat deutlich gemacht, dass solch eine Deutung aufgrund der politischen Auseinandersetzungen zwischen den Kommunen und dem Kaiser nicht möglich sei. ${ }^{123}$ Der kaiserliche Adler wird daher wohl auf die rechtliche Oberhoheit des Imperiums verweisen, die von den Städten der lombardischen Liga nicht in Frage gestellt wurde. ${ }^{124}$ Eventuell wurde der Adler ,auch primär als ein heraldisches Symbol für Recht und Gerechtigkeit verstanden. “125 Der Adler wurde bewusst nicht als Skulptur ausgeführt, sondern nur gemalt, um seiner Präsenz in Bezug auf den Reiter weniger Raum zu geben. Diese wird deutlich durch die Wahl des Materials. Eine Malerei im Außenbereich weist eine deutlich geringere Dauerhaftigkeit auf als die Skulptur aus Marmor. Hierdurch wird der Wille nach Dauerhaftigkeit dieser Kommune ausgedrückt. Dennoch ist der kaiserliche Adler über dem Reiter positioniert und somit wird die Macht des Kaisers anerkannt.

Dem zeitgenössischen Betrachter wäre wahrscheinlich auch ohne die Inschrift klar gewesen, dass mit dem Bildnis der Podestà gemeint ist, da es sich am Kommunalpalast befand. So hat das Bildnis auch eine Wirkung auf Leseunkundige. Der Lesekundige kann erkennen, dass die Inschrift eindeutig auf die memoria des Dargestellten abzielt. ${ }^{126}$ Sie ist ein Erinnerungszeichen an den Podestà und seine Taten. Diesen Bezug konnten aber nur die verstehen, die lesen konnten, oder Zeitgenossen, die die Bauarbeiten erlebt hatten, ${ }^{127}$ oder auch Personen, denen der Inhalt und die Zusammenhänge vermittelt wurden. Die Inschrift richtet sich also nicht nur an Lesekundige, sondern an alle Bürger der Kommune, aber auch an Gäste und Besucher aus Stadt und Land.

122 Liebenwein 1983, 119. Auch Valentiner brachte das Reiterstandbild mit der politischen Kunst Kaiser Friedrichs II. in Verbindung. Siehe Valentiner 1956, 136.

123 Seiler 1989, 73-75.

124 Ebd., $74 f$.

125 Ebd., 75.

126 Nach Lomartire unterstützt das metrische Versmaß und die eloquente Ausdrucksweise dieses Vorhaben. Siehe Lomartire 2015, 107.

127 von Hülsen-Esch 1994, 25. 


\section{Die Inschriftentafel an der Porta di San Bartolomeo in Montefalco}

An der Porta di San Bartolomeo, heute auch Porta di Federico II genannt, am südlichen Teil der Stadtmauer von Montefalco in der Provinz Perugia in Umbrien ist eine Inschriftentafel mit Bau- und Künstlerinschrift auf der stadtauswärts liegenden Seite angebracht (Abb. 9 und 10). Vom Portal aus hat man einen Blick nach Südosten in das Tal, das in Richtung Spoleto führt. Meistens sind Inschriftentafeln dieser Art nicht verziert. In diesem Fall wurde versucht, eine ausgewogene Komposition zu finden, in der dennoch die Inschrift dominierend erscheint. Die Tafel befindet sich zwischen dem Scheitel des spitzbogigen Portalbogens und dem abschließenden Gesims des Stadttores.

Die Tafel befand sich Anfang des 20. Jahrhunderts in der Sakristei von S. Francesco in Montefalco ${ }^{128}$ und wurde später wieder an ihrem ursprünglichen Standort, wohl anlässlich einer Restaurierung des Stadttores, vermauert. ${ }^{129}$

Der Rahmen ist bei der Tafel oben breiter als an den anderen Seiten, um einen Großteil der Inschrift in zwei Zeilen aufzunehmen. In der Mitte der oberen Rahmenleiste wurde ein gleicharmiges Kreuz, ein griechisches Kreuz, herausgearbeitet, indem zwischen den Armen ein Kreis vertieft wurde. Die Kreuzarme verbreitern sich etwas nach außen zum Kreis hin. Die Kreuzmitte weist einen Ring ähnlich wie bei einem keltischen Kreuz auf. Am Ende der Inschrift ist bündig mit der inneren Kante der Rahmenleiste, nahe dem rechten Tafelrand, ein quadratisches Loch eingelassen, dessen Funktion bisher nicht geklärt werden konnte. Es könnte sich um eine spätere Montierungsvorrichtung handeln oder um eine zeitgenössische, um einen Stab anzubringen, der zur Aufnahme von Fahnen etc. gedient haben könnte. Eine andere Möglichkeit wäre eine technische Funktion des Loches, eventuell vor der Ausarbeitung der Tafel. Zum Transport des Steines auf der Baustelle wird es nicht gedient haben, da hierzu zu dieser Zeit meistens sogenannte Wolfslöcher Verwendung fanden. Jedenfalls nimmt das Loch Rücksicht auf die Inschrift oder die Inschrift auf das Loch. Das vertiefte Tafelfeld ist durch vier diagonale Stege gegliedert. Hierdurch entstehen fünf Dreiecksfelder, wobei die beiden Äußeren im Vergleich zu den anderen durch die Rahmenbegrenzung vertikal geteilt sind und nur aus einer Hälfte bestehen. Man könnte sich also einen fortlaufenden Fries mit stehenden und kopfstehenden gleichseitigen Dreiecken vorstellen, bei dem die beiden äußeren stehenden Dreiecke zur Hälfte abgeschnitten sind. Das mittlere stehende Dreieck nimmt das Kreuz aus der oberen Rahmenleiste, nun aber größer, wieder auf. In einem vertieften Kreis ist durch Stege

128 Urbini 1913, 112. Angelini Rota führt das Relief in der Kirche San Francesco auf, die schon damals als Museum benutzt wurde. Siehe Angelini Rota 1920, 197.

129 Campana 1972, 157. Das Datum hierzu ist nicht bekannt. Dietl vermutet vor 1972, da der Wappenstein im Katalog von Nessi/Scarpellini 1972 nicht mehr aufgeführt ist. Siehe Dietl 2009, Bd. 2, 1077. 


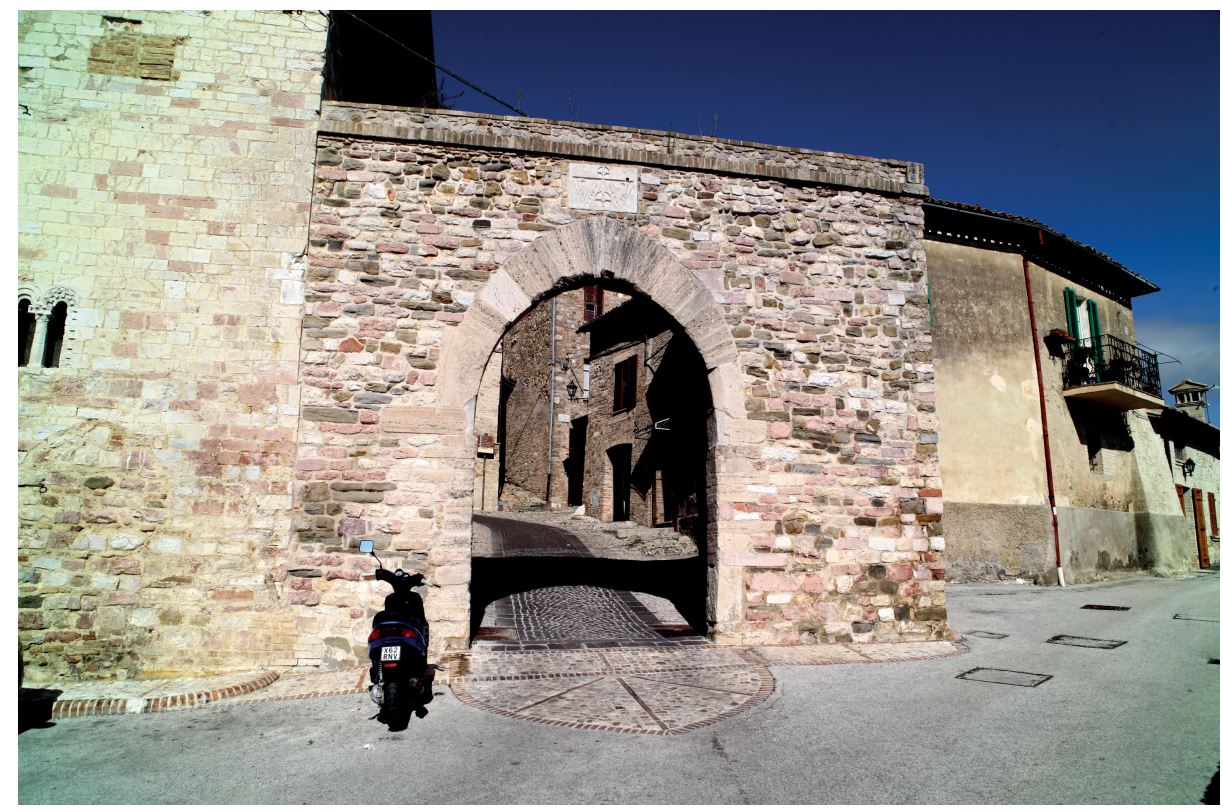

Abb. 9: Montefalco, Porta di San Bartolomeo (Foto @ Wilfried E. Keil).

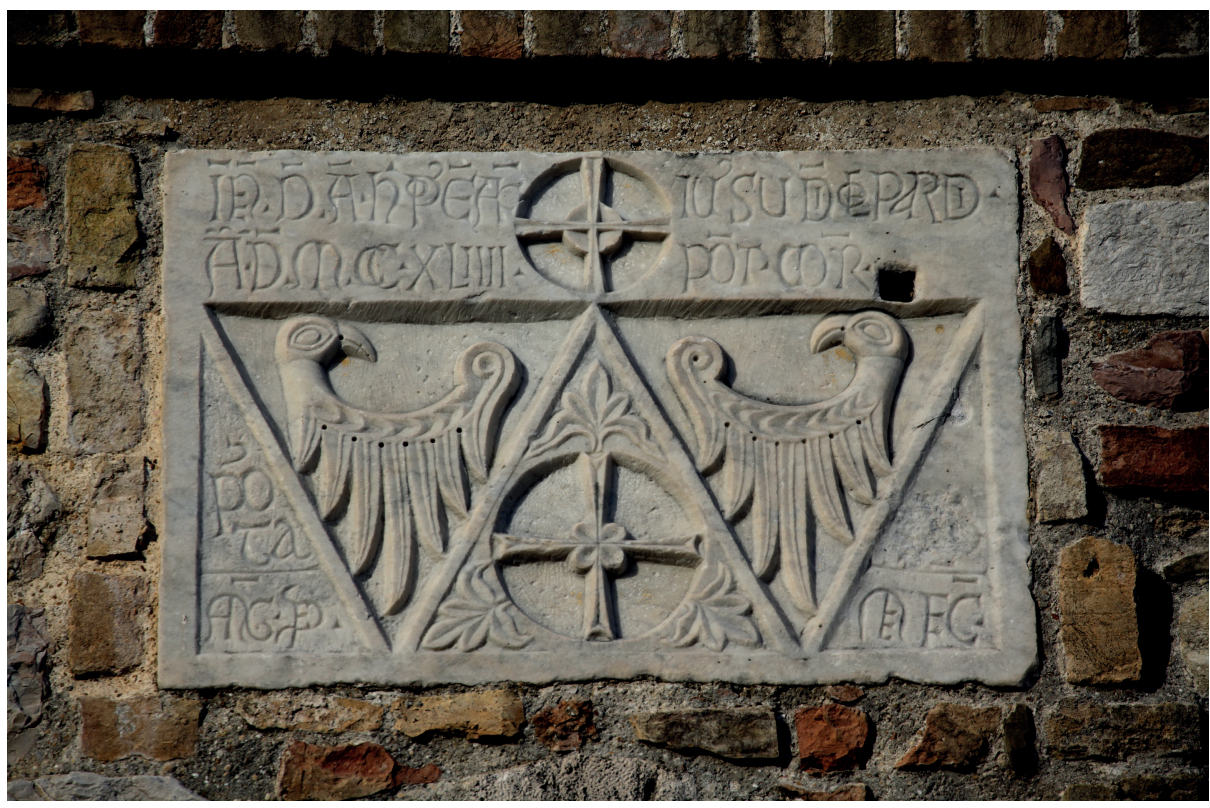

Abb. 10: Montefalco, Porta di San Bartolomeo, Inschrift (Foto @ Wilfried E. Keil). 
ein gleicharmiges Kreuz herausgearbeitet. Die eingekerbten Kreuzarme verbreitern sich zu den Enden hin, die halbkreisförmig eingezogen sind. In den Ecken der Kreuzmitte sind kleine Blätter eingestellt. Vom Kreuzmedaillon vermitteln fünffingrige Blätter zu den drei Spitzen des Dreiecks. Die beiden auf dem Kopf stehenden Dreiecke „beherbergen“ jeweils einen Adlerkopf mit einer zum Inneren gerichteten Schwinge. Die Federn der Schwingen sind stilisiert. Die Adler geben also ein verkürztes Bildnis eines Adlers wieder. Beide sind gegenständig angeordnet, haben ihre Köpfe nach innen gedreht und scheinen sich daher anzublicken. Bei den Adlern wird es sich um das kaiserliche Wappentier handeln. ${ }^{130}$ Die Inschriftentafel ist also zugleich Wappentafel. Die beiden halben stehenden Dreiecke bilden Schriftfelder.

Die Inschriften sind in einer frühgotischen Majuskel ${ }^{131}$ geschrieben und haben Worttrenner in Punktform.

Bei der Inschrift auf der Rahmenleiste sind die beiden Zeilen links des Kreuzes zuerst zu lesen und dann die beiden rechts des Kreuzes. Die Inschrift auf der oberen Rahmenleiste lautet:

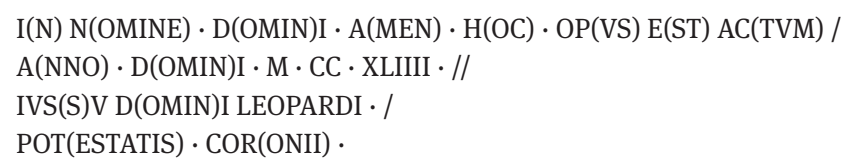

Im Namen des Herrn Amen. Dieses Werk wurde ausgeführt im Jahr des Herrn 1244 auf Befehl des Herrn Leopardus, des Podestà von Coccorone. ${ }^{132}$

130 Dietl 2009, Bd. 2, 1078. Es konnten keine Hinweise für den Gebrauch eines anderen Wappens mit Adlern in Montefalco gefunden werden. Eine Deutung als Falken vom Namen der Stadt her ist auszuschließen, da die Stadt damals einen anderen Namen hatte. Sie ist in den Quellen als Coronium, Castrum Coronii oder Coccoronium belegt. Siehe Nessi 1977, 39 Anm. 77. Zu dem alten Namen der Stadt siehe auch Urbini 1913, 87. Die Raubvögel wurden in der Forschung bisher immer als Adler identifiziert und die Tafel als imperiale Wappentafel. Siehe besonders Urbini 1913, 112; Angelini Rota 1920, 187; Spagnesi 1972, 49; de Vecchi Ranieri 1996, 63f.; Montefalco 1997, 9; Orazi 2011, 45. Zu einer angeblich typischen geometrischen Konstruktion des imperialen Adlers, die Gianfranco Spagnesi auf Grundlage des Stadtplans von Aquila entwickelt, siehe Spagnesi 1972, bes. 46f. (inklusive schematischer Zeichnungen). Diese wendet Spagnesi nicht nur auf Münzen an, sondern auch auf das Relief von Montefalco. Hierzu verwendet er allerdings auch eine leicht verzerrte Photographie. Siehe ebd., 49 u. Abb. 1 und 2. Maila Orazi sieht in der Darstellung der imperialen Adler und der beiden Kreuze einen Hinweis auf die schweren Zeiten für die Stadt in der Epoche des Kampfes zwischen Imperium und Papsttum. Siehe Orazi 2011, 45.

131 Augusto Campana bezeichnet die Schrift als gotische Majuskel und Dietl als romanische Majuskel. Siehe Campana 1972, 159; Dietl 2009, Bd. 2, 1078. In der Tat erinnern einige Formen eher noch an eine romanische Majuskel, aber andere, wie vor allem die Form des $\mathrm{M}$ und die weiteren runden Formen weisen auf eine gotische Majuskel hin.

132 Zur Inschrift siehe Dietl 2009, Bd. 2, 1078. Campana löste die erste Zeile der Inschrift anders als Dietl auf: I(M)PE(NSIS?) D(OMINI) A(...) H(OC) OP(VS) E(ST) AC(TVM). Den letzten Namen in der Inschrift löste er als COR(TONE) oder COR(NETI) auf. Siehe Campana 1972, 157f. Dietl hat diese Lesarten 
Die Inschrift des linken und rechten Dreiecks bilden gemeinsam zwei inhaltlich zueinander gehörende Zeilen. Es muss also über die Grenzen der Dreiecke hinweg gelesen werden. Dies wird durch eine Ritzlinie zwischen den beiden Zeilen verdeutlicht. Die erste Zeile ist hierbei eigentlich in zwei Zeilen aufgeteilt.

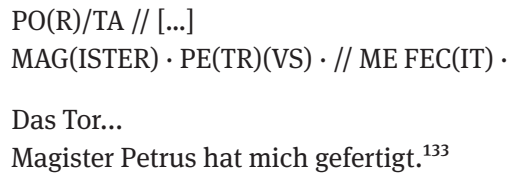

Die Inschrift auf der oberen Rahmenleiste weist extrem viele Kürzungen auf, die meistens durch Kürzungszeichen gekennzeichnet sind. Zudem sind viele Enklaven und Verschränkungen verwendet worden. Ein derartig starkes Abkürzen ist aus der Antike nicht bekannt. Bei der Inschrift in den Dreiecken sind die Wörter der letzten Zeile ebenfalls stark abgekürzt und die Buchstaben durch Enklaven und Verschränkungen nahezu verschleiert. So besteht das Magister sowohl aus einer Enklave als auch einer Verschränkung und einem Kürzungszeichen. Der Name des Portals ist nicht mehr ersichtlich, da die entsprechende Inschrift ausgemeißelt wurde. ${ }^{134}$ Durch die starken Kürzungen ist die Lesbarkeit der Inschrift extrem erschwert. Auch die heutigen Wissenschaftler hatten ihre Mühe damit, die Inschrift entsprechend zu entziffern. Aber vielleicht ging es gar nicht darum, dass ein Passant die Inschrift lesen konnte. Wenn man vor dem Tor steht ist sie für jeden gut sichtbar. Für einen nicht Lesekundigen bzw. einen, der die komplexen Kürzungen nicht auflösen konnte, war die beigefügte Skulptur entscheidend und die Tatsache, dass etwas verschriftlicht war. Durch die Platzierung und die Kürzungen der Inschrift wird eindeutig klar, dass die Inschrift

überzeugend zurückgewiesen. Siehe Dietl 2009, Bd. 2, 1078. Zu den paläographischen Details der Inschrift siehe Campana 1972, 159.

133 Zur Inschrift siehe Dietl 2009, Bd. 2, 1078.

134 Dietl schreibt, dass „rechts deutlich die Spuren einer Rasur zu sehen sind.“ Siehe ebd. Dem kann nach eigener Anschauung nur zugestimmt werden. Silvestro Nessi hatte die Inschrift ohne Begründung mit HAEC EST ACTA ergänzt. Siehe Nessi 1971a, 27; Nessi 1971b, 115. Campana fragt sich, ob die Buchstaben absichtlich getilgt worden seien. Er vermutet drei Buchstaben mit einem Kürzungszeichen, eventuell in Form eines Omega. Der zweite Buchstabe könnte für ihn ein E, L oder C sein. Es könnte die Abkürzung von $\mathrm{S}(\mathrm{AN}) \mathrm{C}(\mathrm{T}) \mathrm{O}$ sein, aber er ist selbst nicht davon überzeugt. Er stellt die Hypothese auf, dass die Rasur eventuell stattgefunden hat, da man bemerkte, dass kein Platz mehr da war, um den Namen des Portals vollständig unterzubringen. Weiter oben im Feld ist ein Zeichen, das für ihn ein O sein könnte. Siehe Campana 1972, 159. Die Hypothese mit der Rasur erscheint recht unwahrscheinlich, wenn man den Rest der Inschrift mit seinen Kürzungen betrachtet. Dietl folgt Campana in Teilen und vermutet „ursprünglich wohl drei Buchstaben mit omegaförmigem Kürzungszeichen und hochgestelltem O“. Siehe Dietl 2009, Bd. 2, 1078. Ein Stadtführer, der von der Accademia di Montefalco per la Storia, l'Arte e la Cultura Locale verfasst wurde, übernimmt die Lesung von Dietl, aber löst stattdessen MAG(ISTER) als ANG(ELUS) auf. Siehe Montefalco 1997. 
dem Relief in der Wichtigkeit untergeordnet war. Die Tiefe des Reliefs ist größer als die der Inschrift, was die Sichtbarkeit deutlich erhöht. Die wichtigen Informationen wie die Jahreszahl und der Name des herrschenden Podestà sind trotz der Abkürzungen deutlich lesbar.

Durch die Adler war ein imperialer Bezug hergestellt, der für den Betrachter ersichtlich war. ${ }^{135}$ Man kann durch eine imperiale Inschrift an dieser Stelle schließen, dass es sich entweder um eine Bauinschrift oder eine Inschrift handelt, die den Kaiser als Protektor der Stadt nennt bzw. in der sich die Stadt in dieser Zeit als kaisertreu bekennt. Für den Ankommenden war also klar, dass er mit dem Durchschreiten des Portals einen „kaisertreuen Raum“ betrat. Albert Dietl vermutet in der Signatur des Magister Petrus, die im linken Dreieck unten beginnt und im rechten weitergeführt wird, eher den Bildhauer des Reliefs als den Architekten des Stadttores. ${ }^{136}$

Das Tor ist das südliche Stadttor von insgesamt fünf Stadttoren und wurde nach der daran liegenden, erstmals 1219 erwähnten Kirche S. Bartolomeo genannt. Die Kirche wurde bereits im 12. Jahrhundert erbaut. ${ }^{137}$ Das Tor der Stadtmauer des 12. Jahrhunderts wird erstmals 1233 erwähnt. ${ }^{138}$ Wegen Stadterweiterungen im 13. Jahrhundert wurde die Stadtmauer um einen zweiten größeren Mauerring ergänzt, der genau an der Porta di S. Bartolomelo an die ältere Stadtmauer stieß. In diesem Zusammenhang wurde wohl das Stadttor 1244 im kommunalen Auftrag erneuert und mit der Tafel versehen. ${ }^{139}$ Die Inschrift nennt daher den Auftraggeber der Erweiterung, Herrn Leopardus, den Podestà von Coccorone, ${ }^{140}$ den früheren Namen von Montefalco, das Jahr 1244 und den Erbauer des Portals.

Im Streit zwischen den Ghibellinen, den Kaisertreuen, und den Guelfen, den Papsttreuen, war Anfang 1240 die Stadt Coccorone unter Capitano Tommaso d'Aquino, Conte di Acerra, mit den umbrischen Nachbarstädten Spello, Bevagna, Bettona und Trevi zu Kaiser Friedrich II. (1212-1250) übergegangen, der vom 9. bis 13. Februar in der Stadt weilte. ${ }^{141}$ Nach der Exkommunikation Friedrichs II. am 17. Juli 1247 auf dem Konzil von Lyon wandte sich die Kommune von Friedrich ab und änderte nach der

$135 \mathrm{Zu}$ Adlern in der Bauskulptur als imperiale Zeichen an Herrschaftsbauten im Mittelalter siehe Rödel 2016.

136 Dietl 2009, Bd. 2, 1078. Maila Orazi sieht in Magister Petrus ebenfalls den Bildhauer. Siehe Orazi 2011, 45.

137 Dietl 2009, Bd. 2, 1079.

138 Chiuini 1980, 202 Anm. 3.

139 Chiuini 1980, 204; Nessi 1971a, 27; Nessi 1977, 87; Nessi 2001, 33. Zur Stadtentwicklung und der Stadtmauererweiterung zur Zeit Friedrichs II. im Allgemeinen siehe Nessi 2001. Der Graf von Spoleto nahm in der territorialen Abgrenzung der Ghibellinen von den Guelfen eine entscheidende Rolle ein und somit auch Montefalco, wo, wie in anderen Städten, kaiserliche Truppen stationiert waren. Siehe Chiuini 1980, 204. Zur Situation des Grafen von Spoleto zwischen Papst und Kaiser zu Zeiten Friedrichs II. siehe ausführlich Nessi 1983.

140 Zum Auftraggeber siehe auch Nessi 1977, 87.

141 Nessi 1983, $919 f$. 
Zerstörung der Stadt durch die kaiserlichen Truppen ihren Namen in Montefalco, der 1249 erstmals urkundlich belegt ist. ${ }^{142}$

Der Name des Portals im oberen Teil des rechten Dreiecks wurde daher wohl in späterer Zeit getilgt. Dies hängt wohl mit dem früheren Namen des Portals zusammen. Die Stadt hatte sich vom Kaiser abgewandt und so musste wohl eine Inschrift, die auf den Herrscher verwies, weichen. Es fragt sich nur, wieso man die Adler, die kaiserlichen Wappentiere, nicht ebenfalls abschlug, da diese eigentlich noch mehr für den Kaiser standen und auch für Leseunkundige zu verstehen waren. Ein Grund hierfür könnte sein, dass man dadurch nahezu die Hälfte der Tafel abgeschlagen hätte und die kaiserlichen Wappentiere nicht mit einem speziellen Kaiser in Verbindung gebracht werden können.

Die Gestaltung der Inschrift und der Skulptur bilden hier also eine noch größere Einheit als bei den beiden anderen Beispielen. Hier sind sie aus einem Stein gehauen. Der Inschrift kommt, wie bereits erwähnt, durch Platzierung und Kürzungen in der Tafel eine untergeordnete Rolle zu. Die Fehlstelle verweist auf etwas, was durch Zerstörung nicht mehr in der Inschrift steht oder vielleicht sogar niemals stand.

\section{Unterschiedliche Formen der Korrelationen zwischen Schrift und Bild}

Es sind also unterschiedliche Arten und unterschiedliche Gewichtungen von Schriftund Bildverhältnissen zu finden. Eine spätere Zusammenstellung einer Schrifttafel und eines Bildnisses kann nicht nur ein Bildnis uminterpretieren, sondern auch die Schrift beeinflussen. Im Fall von Genua hat die Löwenskulptur die Sichtbarkeit der Inschrift reduziert und somit die Präsenz der Schrift eingeschränkt. Aber der Löwenkopf kann auch ikonographisch für diesen Standort und zum Inhalt der Inschriftentafel umgedeutet werden, wie gezeigt werden konnte. Sowohl die Inschriftentafel als auch die Skulptur haben, bevor sie zusammengeführt wurden, auch alleine gewirkt.

Das Reiterstandbild des Oldrado da Tresseno würde auf Grund der Darstellungstradition auch ohne die Inschrift seine Wirkung als Herrschaftssymnbol haben. Aber die Inschrift gibt dem Betrachter zusätzliche entscheidende Informationen, den Namen und die Taten des Podestà. Die Inschrift könnte auch alleine für sich stehen, allerdings würde sie ohne eine Skulptur bei dieser Größe nicht so auffallen und dadurch an Präsenz verlieren.

Bei der Inschriften- und Wappentafel in Montefalco bilden Schrift und Bild eine starke Einheit. Durch die Ausgestaltung der Tafel wird die Inschrift zu einem

142 Urkundlicher Beleg: Urbini 1913, 87. Zu den Legenden zur Namensherkunft von Montefalco siehe Nessi 1983, 929. Siehe hierzu auch Urbini 1913, 87; Chiuini 1980, 205 Anm. 19. 
Blickfang. Sie wurde hierdurch sicherlich häufiger wahrgenommen als eine reine Inschriftentafel. Vor allem Leseunkundige hatten somit einen besseren Zugang zur Tafel und konnten sich hierdurch teilweise wohl an bereits vorgelesene inhaltlich ähnliche Inschriften erinnern. Zudem wurde durch die Adler und Kreuze selbst und auch ihre Platzierung einem Leseunkundigen der Konflikt zwischen Kirche und Staat in Erinnerung gerufen.

Alle drei Beispiele haben noch etwas gemeinsam: Sowohl in Genua als auch in Montefalco spricht durch die Inschrift das Bauwerk zu uns. In Genua steht zweimal ME, einmal bei FIERI ME IVSSIT und dann bei IVSSV(M) M(E) TRA(N)STVLIT und in Montefalco einmal ME bei ME FEC(IT). In Mailand hingegen wird der Betrachter durch SCA(N)DIS direkt angesprochen. Auch hier spricht die Inschrift wieder für das Bauwerk. Die Inschriften kann man also als Akteure sehen und durch sie wiederum die Bauwerke als Akteure. Zudem kann man hierbei noch die Skulpturen mitberücksichtigen, da diese sich entweder direkt auf die Inschrift beziehen oder auf diese beziehen lassen. Für Skulpturen, die Akteure sind bzw. handeln, wurde die Theorie des Bildaktes entwickelt. ${ }^{143}$ Analog hierzu kann man bei den Inschriften von einem Schriftakt sprechen. Wie beim Bildakt das Bildnis, fordert beim Schriftakt die Schrift zu einer Interaktion zwischen menschlichem Akteur und materiellem Artefakt auf. ${ }^{144}$

Allerdings ist bei einer kommunalen Inschrift mit Skulptur der Inhalt der Inschrift in den meisten Fällen zum Verständnis wichtiger als bei Skulpturen mit Inschriften im sakralen Bereich, da sie orts- und zeitgebunden sind und daher einer genaueren Erklärung bedürfen. Im sakralen Bereich kann man bei Skulpturen mit Inschriften häufig von einer weit verbreiteten Kenntnis der biblischen Geschichten ausgehen. Durch das Aufstellen von kommunalen Inschriften mit Skulpturen an zentralen Orten wird die Macht der Kommune den Bürgern und Fremden gezeigt. Die Präsenz der Artefakte im öffentlichen Raum demonstriert ein Selbstverständnis der Kommune. Mit der Wahl des Materials Stein bzw. Marmor wird deutlich, dass auf die Dauerhaftigkeit der Präsenz der Artefakte Wert gelegt wurde, um eine Beständigkeit der Erinnerung zu erlangen. Durch die Skulpturen werden die Wahrnehmung und die Wirkung einer Inschrift erhöht und somit auch eine stärkere Erinnerung gewährleistet.

143 Zum Bildakt siehe besonders Bredekamp 2010.

144 Frese/Keil 2015. 


\section{Literaturverzeichnis}

Annales lanuenses (1926), in: Cesare Imperiale di Sant'Angelo (Hg.), Annali Genovesi di Caffaro e de' suoi continuatori, Bd. 4: Dal 1251 al 1279 (Fonti per la storia d'Italia 14), Rom.

Annales Mediolanenses (1730), in: Ludovico A. Muratori (Hg.), Rerum Italicarum Scriptores ab anno aerae Christianae quingentesimo ad millesimumquingentesimum, Bd. 16, Mailand, 635-840.

Armillotta, Tiziana (2009/10), „Il palazzo conteso. Il Consorzio Autonomo del Porto, la Soprintendenza e la ricostruzione di Palazzo San Giorgio a Genova“, in: Opvs incertvm 6/7, 120-129.

Angelini Rota, Giuseppe (1920), Spoleto e il suo Territorio, Spoleto.

Alizeri, Federico (1847), Guida artistica per la città di Genova, Bd. 2, Genua.

Balke, Thomas E./Keil, Wilfried E./Opdenhoff, Fanny/Stroth, Fabian (2015), „Stein“, in: Thomas Meier, Michael R. Ott u. Rebecca Sauer (Hgg.), Materiale Textkulturen. Konzepte - Materialien Praktiken (Materiale Textkulturen 1), Berlin/München/Boston, 247-267.

Bering, Kunibert (1986), Kunst und Staatsmetaphysik des hochmittelalterlichen Italiens. Zentren der Bau- und Bildpropaganda in der Zeit Friedrichs II. (Kunst. Geschichte und Theorie 5), Essen.

Bocchi, Francesca (1993), „Il Broletto“, in: Milano e la Lombardia in età comunale. Secoli XI-XIII (Katalog der Ausstellung Mailand 1993), Cinisello Balsamo (Mailand), 38-42.

Boerner, Bruno (2008), Bildwirkungen. Die kommunikative Funktion mittelalterlicher Skulpturen, Berlin.

Boito, Carlo (1893), Questioni pratiche di Belle arti, Mailand.

Bredekamp, Horst (2010), Theorie des Bildakts, Berlin.

Calzona, Arturo (2008), Antelami, Parma.

Campana, Augusto (1972), „Nota filologico-critica sulla lapide della porta di S. Bartolomeo a Montefalco presso Spoleto“, in: Gianfranco Spagnesi u. Pier Luigi Properzi (Hgg.), L’Aquila. Problemi di forma e storia della città, Bari, 155-159.

Cavallaro, Luisa (1989), „I cistercensi a Genova: una ,presenza attiva“ intravista presso due cantieri in città nel XIII secolo“, in: Benedictina 36, 329-345.

Cavallaro, Luisa (1992), Il Palazzo del Mare. Il nucleo medioevale di Palazzo San Giorgio, Genua.

Cavallaro, Luisa (1994), „Un cantiere cistercense a Genova: Il Palazzo del Comune“, in: Arte Medievale 8 (1), 153-161.

Cervini, Fulvio (1991), „Sul contributo cistercense all'architettura duecentesca in Liguria“, in: Rivista Cistercense 8, 311-334.

Chiuini, Giovanna (1980), „Montefalco“, in: Federico Zeri (Hg.), Storia dell’arte italiana, Bd. 8: Inchieste su centri minori, Turin, 197-231.

Corio, Bernardino (21554), L'Historia di Milano, Venedig.

Claussen, Peter Cornelius (1994), „Kompensation und Innovation. Zur Denkmalproblematik im 13. Jahrhundert am Beispiel der Reitermonumente in Magdeburg und Bamberg“, in: Herbert Beck u. Kerstin Hengevoss-Dürkop (Hgg.), Studien zur Geschichte der europäischen Skulptur im 12./13. Jahrhundert, Frankfurt a. M., 565-586.

van Cleve, Thomas Curtius (1972), The Emperor Frederick II of Hohenstaufen. Immutator Mundi, Oxford.

Cramer, Thomas (2004), Multivariate Herkunftsanalyse von Marmor auf petrographischer und geochemischer Basis. Das Beispiel kleinasiatischer archaischer, hellenistischer und römischer Marmorobjekte der Berliner Antikensammlung und ihre Zuordnung zu mediterranen und anatolischen Marmorlagerstätten, Diss. Berlin.

Cuneo, Carlo (1842), Memoria sopra l'antico debito pubblico, mutui, compere e Banca di S. Giorgio in Genova, Genua.

Curschmann, Michael (2007), Wort, Bild, Text. Studien zur Medialität des Literarischen in Hochmittelalter und früher Neuzeit (Saecvla spiritalia 43/44), 2 Bde., Baden-Baden. 
Debiais, Vincent (2009), Messages de pierre. La lecture des inscriptions dans la communication médiévale (XIIIe-XIVe siècles) (Culture et société médiévales 17), Turnhout.

Dellacasa, Sabina (Hg.) (1998), I Libri lurium della Repubblica di Genova, Bd. 1.4 (Fonti per la storia della Liguria 11), Rom.

Dietl, Albert (2009), Die Sprache der Signatur. Die mittelalterlichen Künstlerinschriften Italiens. (Italienische Forschungen des Kunsthistorischen Institutes in Florenz 4,6), 4 Bde., Berlin/ München.

Di Fabio, Clario (1995), „Genova, Architettura“, in: Enciclopedia dell’Arte Medievale, Bd. 6, Rom, 500-523.

Dufour Bozzo, Colette (1999), „La querelle sui cistercensi a Genova e in Liguria“, in: Antonio Cadei et al. (Hgg.), Arte d'Occidente. Temi e metodi. Studi in onore di Angiola Maria Romanini, Bd. 1, Rom, 175-185.

Favreau, Robert (1997), Épigraphie médiévale (L’atelier du médiéviste 5), Turnhout.

Ferrando Cabona, Isabella (1998), Palazzo San Giorgio. Pietre, uomini, potere / Stones, Men, and Power (1260-1613), Cinisello Balsamo (Mailand).

Frese, Tobias/Keil, Wilfried E. (2015), „Schriftakte/Bildakte“, in: Thomas Meier, Michael R. Ott u. Rebecca Sauer (Hgg.), Materiale Textkulturen. Konzepte - Materialien - Praktiken (Materiale Textkulturen 1), Berlin/München/Boston, 633-638.

Frese, Tobias/Keil, Wilfried E./Krüger, Kristina (Hgg.) (2014), Verborgen, unsichtbar, unlesbar - zur Problematik restringierter Schriftpräsenz (Materiale Textkulturen 2), Berlin/Boston.

Frugoni, Chiara (1995), „,E vedrà ogni carne la salvezza di Dio“ (LC 3,6): le sculture all'interno del battistero“, in: Chiara Frugoni (Hg.), Benedetto Antelami e il Battistero di Parma, Turin, 109-144.

Galvaneus de la Fiamma (1727), „Manipulus Florum“, in: Rerum Italicarum Scriptores ab anno aerae Christianae quingentesimo ad millesimumquingentesimum, hg. von Ludovico A. Muratori, Bd. 11, Mailand, 531-740.

Giovè Marchioli, Nicoletta (1994), „L'epigrafia comunale cittadina“, in: Paolo Cammarosano (Hg.), Le forme della propaganda politica nel due e nel trecento (Tagung Triest 1993; Collection de l' École Française de Rome 201), Rom, 263-286.

Gramaccini, Norberto (1985), „Die Umwertung der Antike. Zur Rezeption des Marc Aurel in Mittelalter und Renaissance“, in: Herbert Beck u. Peter C. Bol (Hgg.) Natur und Antike in der Renaissance (Katalog der Ausstellung Frankfurt a. M. 1985/1986), Frankfurt a. M., 51-83.

Grandi, Renzo (1988), „Oldrado da Tresseno“, in: Carlo Bertelli (Hg.), Città del vescovo dai Carolingi al Barbarossa, Milano, 240-249.

Grimoldi, Alberto (1983), Il Palazzo della Ragione. I luoghi dell'autorità cittadina nel centro di Milano, Mailand.

Grossi Bianchi, Luciano/Poleggi, Ennio (21987), Una città portuale del Medioevo: Genova nei secoli $X-X V I$, Genova.

Grosso, Orlando (21984), Il Palazzo San Giorgio, Genua.

Gutscher, Daniel (1983), Das Grossmünster in Zürich. Eine baugeschichtliche Monographie (Beiträge zur Kunstgeschichte in der Schweiz 5), Bern.

Haug, Henrike (2016), Annales lanuenses. Orte und Medien des historischen Gedächtnisses im mittelalterlichen Genua (Orbis Mediaevalis 15), Göttingen.

von der Höh, Marc (2006), Erinnerungskultur und frühe Kommune. Formen und Funktionen des Umgangs mit der Vergangenheit im hochmittelalterlichen Pisa (1050-1150), Berlin.

von Hülsen-Esch, Andrea (1994), Romanische Skulptur in Oberitalien als Reflex der kommunalen Entwicklung im 12. Jahrhundert. Untersuchungen zu Mailand und Verona, Berlin. 
Iacobini, Antonio (1997), „Le imposte bronzee del portale maggiore di San Marco. Un riesame dopo il restauro, in: Renato Polacco (Hg.), Storia dell'arte marciana, Bd. 3: Sculture, tesoro, arazzi (Tagung Venedig 1994), Venedig, 267-277.

Jäckel, Dirk (2006), Der Herrscher als Löwe. Ursprung und Gebrauch eines politischen Symbols im Früh- und Hochmittelalter (Beihefte zum Archiv für Kulturgeschichte 60), Köln/Weimar/Wien.

Krause, Karin/Schellewald, Barbara (Hgg.) (2011), Bild und Text im Mittelalter (Sensus. Studien zur mittelalterlichen Kunst 2), Köln/Weimar/Wien.

Keil, Wilfried E. (2014), „Überlegungen zur restringierten Präsenz mittelalterlicher Bauinschriften“, in: Tobias Frese, Wilfried E. Keil u. Kristina Krüger (Hgg.), Verborgen, unsichtbar, unlesbar - zur Problematik restringierter Schriftpräsenz (Materiale Textkulturen 2), Berlin/Boston, 117-142.

Liebenwein, Wolfgang (1983), „Princeps Poetarum. Die mittelalterlichen Vergil-Bilder in Mantua“, in: Viktor Pöschl (Hg.), 2000 Jahre Vergil. Ein Symposium (Tagung Wolfenbüttel 1982; Wolfenbütteler Forschungen 24), Wiesbaden, 109-151.

Lomartire, Saverio (2008), „La statua del Regisole di Pavia e la sua fortuna tra Medioevo a Rinascimento“, in: Joachim Poeschke, Thomas Weigel u. Britta Kusch-Arnold (Hgg.), Praemium Virtutis, Bd. 3: Reiterstandbilder von der Antike bis zum Klassizismus (Symbolische Kommunikation und gesellschaftliche Wertesysteme 22), Münster, 31-73.

Lomartire, Saverio (2015), „,Iustitia, maiestas, curialitas‘. Oldrado da Tresseno e il suo ritratto equestre nel Broletto di Milano“, in: Arte medievale 5, 101-136.

Marcenaro, Mario (1990), „Alfredo D’Andrade“, in: Colette Dufour Bozzo u. Mario Marcenario (Hgg.), Medioevo Demolito. Genova 1860-1940, Genua, 277-311.

Marchesini, Attilio (2008), „Monumento equestre al Podestà Nazaro Ghirardini di Lucca (?)“, in: Arturo Calzona (Hg.), Matilde e il tesoro dei Canossa. Tra castelli e città (Katalog der Ausstellung Reggio Emilia 2008/09), Cinisello Balsamo (Mailand), 558-559.

Mende, Ursula (1981), Die Türzieher des Mittelalters, Berlin.

Montefalco. Guida turistica (1997), Santa Maria degli Angeli (Assisi).

Müller, Rebecca (2002), Sic hostes lanua frangit. Spolien und Trophäen im mittelalterlichen Genua (Marburger Studien zur Kunst- und Kulturgeschichte 5), Weimar.

Müller, Rebecca (2005), „Genova vittoriosa: i trofei bellici“, in: Piero Boccardo u. Clario Di Fabio (Hgg.), Genova e l'Europa mediterranea. Opere, artisti, committenti, collezionisti, Cinisello Balsamo (Mailand), 89-107.

Nelson, Robert S. (2010), „The History of Legends and the Legends of History. The Pilastri Acritani in Venice“, in: Henry Maguire u. Robert S. Nelson (Hgg.), San Marco, Byzantium, and the Myths of Venice (Dumbarton Oaks Byzantine Symposia and Colloquia), Washington, D. C., 63-90.

Nessi, Silvestro (1971a), „Architettura civile medievale dei Monti Martani“, in: Spoletium 13, 25-34.

Nessi, Silvestro (1971b), „Architettura civile medioevale in Umbria nel comprensorio dei Monti Martani“, in: Les Constructions civiles d'intérêt public dans les villes d'Europe au Moyen Âge et sous l'Ancien regime et leur financement (Tagung Spa 1968; Collection Histoire 26), Brüssel, 111-124.

Nessi, Silvestro (1977), Le origini del comune di Montefalco, Spoleto.

Nessi, Silvestro (1983), „Il ducato di Spoleto tra papato e impero al tempo di Federico II“, in: Il Ducato di Spoleto (Tagung Spoleto 1982), Bd. 2, Spoleto, 909-954.

Nessi, Silvestro (2001), „Cinta di mura e sviluppo urbanistico di Montefalco al tempo di Federico II“, in: Boris Ulianich u. Giovanni Vitolo, Castelli e cinte murarie nell'età di Federico II (Tagung Montefalco 1994; Atti di Convegni 7) Rom, 27-33.

Nessi, Silvestro/Scarpellini, Pietro (1972), La chiesa museo di San Francesco a Montefalco, Spoleto. Niewöhner, Philipp (2013/2014), „Byzantine Water Spouts with Zoomorphic Head and Channel“, in: Cahiers Archéologiques. Fin de l'Antiquité et Moyen Âge 55, 79-90. 
Niewöhner, Philipp (2016), „Zoomorphic Rainwater Spouts“, in: Brooke Shilling u. Paul Stephenson (Hgg.), Fountains and Water Culture in Byzantium, Cambridge, 163-181.

Orazi, Maila (2011), Montefalco. Città d'Arte, Perugia.

Paribeni, Andrea (2009), „Le porte ageminate della basilica di S. Marco a Venezia tra storia e committenza“, in: Antonio lacobini (Hg.), Le porte del paradiso. Arte e tecnologia bizantina tra Italia e Mediterraneo (Tagung Rom 2006), Rom, 300-317.

Paul, Jürgen (1963), Die mittelalterlichen Kommunalpaläste in Italien, Diss. Freiburg i. Br.

Polonio Felloni, Valeria (1996), „Da ,opere“ a pubblica magistratura. La cura della cattedrale e del porto nella Genova medievale“, in: Margaret Haines u. Lucio Ricetti (Hgg.), Opera. Carattere e ruolo delle fabbriche cittadine fino all'inizio dell'Età Moderna (Tagung Florenz 1991; Villa I Tatti. The Harvard University Center for Italian Renaissance Studies 13), Florenz, 117-136.

Polonio Felloni, Valeria (1998), „I cistercensi in Liguria (secoli XII-XIV)“, in: Colette Bozzo Dufour u. Anna Dagnino (Hgg.), Monasteria Nova. Storia e architettura dei cistercensi in Liguria (Secoli XII-XIV), Genua, 3-78.

Reinle, Adolf (1969), „Der Reiter am Züricher Grossmünster“, in: Zeitschrift für Schweizerische Archäologie und Kunstgeschichte 26, 21-46.

Remondini, Marcello (1874), „Iscrizioni medievali della Liguria“, in: Atti della Società Ligure di Storia Patria 12, 1-116.

Rödel, Volker (2016), „Zur Adlersymbolik an und in Herrschaftsbauten des Mittelalters“, in: Tiere auf Burgen und frühen Schlössern. Forschungen zu Burgen und Schlössern 16, 65-75.

Rotondi Terminiello, Giovanna (1977), Palazzo San Giorgio (Guide di Genova 38), Genua.

Saletti, Cesare (1997), Il Regisole di Pavia, Como.

Salimbene de Adam (1998), Cronica, 2 Bde. (Corpus Christianorum Continuatio Mediaeualis 125), hg. von Giuseppe Scalia, Turnhout.

Saurma-Jeltsch, Liselotte E. (1988), „Textaneignung in der Bildersprache. Zum Verhältnis von Bild und Text am Beispiel spätmittelalterlicher Buchillustration“, in: Wiener Jahrbuch für Kunstgeschichte 41, 41-59 u. 173-184.

Seiler, Peter (1989), Mittelalterliche Reitermonumente in Italien. Studien zu personalen Monumentsetzungen in den italienischen Kommunen und Signorien des 13. und 14. Jahrhunderts, Diss. Heidelberg.

Silva, Augusta (Hg.) (1987), Corpus Inscriptionum Medii Aevi Liguriae, Bd. 3: Genova. Centro Storico, Genua.

Späth, Markus (2011), „Bild, Schrift und Sprache. Überlegungen zur Intermedialität in der italienischen Bauplastik des 12. Jahrhunderts am Beispiel der Westfassade von San Clemente a Casauria“, in: Karin Krause u. Barbara Schellewald (Hgg.), Bild und Text im Mittelalter (Sensus. Studien zur mittelalterlichen Kunst 2), Köln/Weimar/Wien, 125-151.

Spagnesi, Gianfranco (1972), „L’Aquila: Problemi di forma e storia della città“, in: Gianfranco Spagnesi u. Pier Luigi Properzi (Hgg.), L’Aquila. Problemi di forma e storia della città, Bari, 17-84.

Toesca, Pietro (1927), Storia dell'Arte italiana II. Il Medioevo, Turin.

Untermann, Matthias (2001), Forma Ordinis. Die mittelalterliche Baukunst der Zisterzienser, München/Berlin.

Urbini, Giulio (1913), Spello, Bevagna, Montefalco (Collezione di monografie illustrate. Italia artistica 71), Bergamo.

Valentiner, Wilhelm Reinhold (1956), The Bamberg Rider. Studies of Medieval German Sculpture, Los Angeles.

de Vecchi Ranieri, Marilena (Hg.) (1996), Montefalco. I luoghi, gli itinerari, la storia, Città di Castello. Venturi, Adolfo (1904), Storia dell'arte italiana, Bd. 3: L'arte romanica, Mailand. 
Verzar, Christine B. (1994), „Text und Bild in der norditalienischen Romanik: Skulpturen, Inschriften, Betrachter“, in: Beck, Herbert/ Hengevoss-Dürkop, Kerstin (Hgg.), Studien zur Geschichte der europäischen Skulptur im 12./13. Jahrhundert, Frankfurt am Main, 495-504.

Vio, Ettore (2009), „Le porte bizantine di Venezia: storia e restauro“, in: Antonio lacobini (Hg.), Le porte del paradiso. Arte e tecnologia bizantina tra Italia e Mediterraneo (Tagung Rom 2006), Rom, 283-300.

Weigel, Thomas (1996), „Spolien und Buntmarmor im Urteil mittelalterlicher Autoren“, in: Joachim Poeschke (Hg.), Antike Spolien in der Architektur des Mittelalters und der Renaissance, München, 117-153.

Woelk, Moritz (1995), Benedetto Antelami: die Werke in Parma und Fidenza, Münster. 